\title{
Application-Based Production and Testing of a Core-Sheath Fiber Strain Sensor for Wearable Electronics: Feasibility Study of Using the Sensors in Measuring Tri-Axial Trunk Motion Angles
}

\author{
Ahmad Rezaei ${ }^{\dagger}$, Tyler J. Cuthbert ${ }^{\dagger}$, Mohsen Gholami and Carlo Menon *D \\ Menrva Research Group, Schools of Mechatronic Systems \& Engineering Science, Simon Fraser University, \\ Metro Vancouver, BC V5A1S6, Canada; ahmad_rezaei@sfu.ca (A.R.); cuthbert@sfu.ca (T.J.C.); \\ mohsen_gholami@sfu.ca (M.G.) \\ * Correspondence: cmenon@sfu.ca \\ + The two authors contributed equally to this paper.
}

Received: 20 August 2019; Accepted: 30 September 2019; Published: 3 October 2019

check for updates

\begin{abstract}
Wearable electronics are recognized as a vital tool for gathering in situ kinematic information of human body movements. In this paper, we describe the production of a core-sheath fiber strain sensor from readily available materials in a one-step dip-coating process, and demonstrate the development of a smart sleeveless shirt for measuring the kinematic angles of the trunk relative to the pelvis in complicated three-dimensional movements. The sensor's piezoresistive properties and characteristics were studied with respect to the type of core material used. Sensor performance was optimized by straining above the intended working region to increase the consistency and accuracy of the piezoresistive sensor. The accuracy of the sensor when tracking random movements was tested using a rigorous 4-h random wave pattern to mimic what would be required for satisfactory use in prototype devices. By processing the raw signal with a machine learning algorithm, we were able to track a strain of random wave patterns to a normalized root mean square error of $1.6 \%$, highlighting the consistency and reproducible behavior of the relatively simple sensor. Then, we evaluated the performance of these sensors in a prototype motion capture shirt, in a study with 12 participants performing a set of eight different types of uniaxial and multiaxial movements. A machine learning random forest regressor model estimated the trunk flexion, lateral bending, and rotation angles with errors of $4.26^{\circ}, 3.53^{\circ}$, and $3.44^{\circ}$ respectively. These results demonstrate the feasibility of using smart textiles for capturing complicated movements and a solution for the real-time monitoring of daily activities.
\end{abstract}

Keywords: fiber; strain sensor; core-sheath; piezoresistive; resistive sensor; random wave pattern; machine learning; trunk motion tracking; wearable sensors; smart shirt; random forest regressor

\section{Introduction}

Wearable electronics have become increasingly popular with the advancement of materials and electronics over the past two decades, producing more flexible and integrated functional materials. Wearable electronics span a wide variety of applications such as biomedical monitoring (i.e., body vitals), motion tracking, and integrated personal electronics [1]. Electronic components have become increasingly smaller, and have been designed for incorporation into wearable devices in fiber morphologies, including applications in energy harvesters [2-6], energy storage [2,7-10], and sensors [1,11]. Fiber sensors have been realized for strain [12-14], pressure [15-17], and chemical monitoring [18]. While there are non-wearable technologies that are able to track movement accurately 
(i.e., electromagnetic tracking and optoelectronic motion capture [19]), they have limitations that restrict their application in the workplace environment (sophisticated hardware, high cost, non-portable, limited capture space). Flexible fiber strain sensors have the potential for seamless integration into textiles and clothing creating wearable systems for in situ tracking of movements and further quantitative physical exposure measurement for injury risk assessment, with increased mobility and comfort, and without spatial restrictions—such as those with motion capture systems.

Besides flexible fiber sensors, inertial measurement units (IMU) have been a popular wearable alternative to alleviate the limitations of non-wearable systems. IMU sensors are microelectromechanical sensors consisting of an accelerometer, gyroscope, and magnetometer, which are able to capture the orientation of the sensor with respect to the earth-fixed reference coordinate system [20]. Several IMU units can be used together to form a wearable inertial motion capture (IMC) system. Recently, IMC systems have been used in several kinematic information measurement applications, tracking the lower extremities [21,22], spine [23,24], and trunk [25]. However, important performance limitations of IMCs have been reported $[26,27]$. The IMC performance accuracy was shown to decrease significantly by the movement characteristics (movement complexity, range, speed, and period) [20,26-29]. Fast complex movements over long periods of time have higher errors compared to short simple tasks $[27,30]$. In addition, magnetometers are extremely sensitive to environmental ferromagnetic disturbances and cause a considerable amount of error in IMU heading estimation [31,32]. These limitations have hindered the more widespread use of IMC systems in three-dimensional (3D) motion tracking, and therefore, the application of smart textiles employing flexible fiber strain sensors has been investigated as a substitute solution.

Flexible fiber strain sensors are commonly built on conductive components (i.e., metals [13,33], graphene [34,35], carbon nanotubes [36], carbon black [37], polymers [38,39], and ionic liquids [40,41]) and flexible polymers or substrates, as either composites or layered structures. There are a number of current methods to create layered coaxial fibers-with up to four layers-including melt spinning/extrusion [42], wet spinning [43,44], electrospinning [45,46], and newer technologies such as pressurized gyration spinning [47,48], solution blowing [49], and the use of 3D printers [50]. The advancement of manufacturing precise multilayered fibers will inevitably lead to more advanced multifunctional materials.

Thus far, fiber sensors have been produced using either resistive or capacitive sensing mechanisms $[1,11,51]$. Resistive sensors are attractive because of their low cost, low power consumption, ease of production, and non-specialized signal processing [52]. Piezoresistive sensors that utilize a polymer composite rely on the disconnection-reconnection of conductive pathways within the material when strain is applied [52]. This destructive mechanism often results in signal drift (i.e., an increase in the baseline resistance) and changing gauge factors, since conductive pathway reconnection may not occur. There have been a few recent examples using rubber elastic support core materials with piezoresistive sheaths to create high-performance fiber sensors. The piezoresistive sheaths have been comprised of carbon nanotube forests [36,53], embedded graphene nanoplatelets [54], silver nanowires/poly(vinylidenefluoride-co-trifluoroethylene) [55], carbon nanotube-silicone rubber composites [44], and elastomer-wrapped carbon nanotube fibers [56]. However, the effect of the core material properties on sensor performance has yet to be studied for piezoresistive thermoplastic elastomer polymer composites.

Thus far, accurately tracking three-dimensional (3D) kinematic motion beyond proof-of-concept testing using fiber strain sensors has been limited. Reports of kinematic tracking using piezoresistive sensors have targeted the wrist, hand, trunk, and knee gait $[37,57,58]$, as well as a full body system. The potential of smart sensing textiles for angle measurement in planar movements and joints with one degree-of-freedom such as knee and fingers has been well established. However, the feasibility of these systems for angle measurement in complex multiplanar movements has yet to be evaluated. Bridging the gap between new sensor production and larger cohort studies researching accuracy and applicability is required to allow this field to flourish. Indeed, testing sensors using strains and 
strain rates that mimic what prototype devices would be ultimately tracking allows screening and optimization prior to creating these devices to ensure that sensor performance will be satisfactory. Sensor performance metrics beyond basic characterization are also important. For instance, testing scenarios that indicate how well a sensor will perform when exposed to real-world random movements is beneficial so that the applied research can focus on other factors such as sensor placement, the number of sensors required, and the signal processing to obtain the optimal and the highest quality kinematic motion information possible.

Herein, we outline the fabrication, characterization, and application-based optimization of core-sheath strain sensors with readily available starting materials that are easy to produce, and amenable to current fiber/textile production methods; then, we utilize this sensor to develop a smart shirt for accurately measuring the three-dimensional kinematic angles of the trunk.

The sensor was fabricated using two cores (a thermoplastic elastomer and an elastic core) that were hypothesized to support the sensing piezoresistive sheath and negate the negative effects of the addition of the conductive additives (i.e., lower elasticity, higher rigidity). In this case, we chose to target the motion tracking of the lower back angles that would be associated with a prototype device, allowing the optimization of the sensor for the strains associated with this location of the body. This type of specific application was intended to highlight that although many reports of new fabrication methodologies for fiber-based sensors are being reported, quite often, the actual performance of those sensors in a tracking application may not be sufficiently accurate. We used a two-prong approach. First, we determined the limits of the materials we were using with respect to their mechanical and piezoresistive properties. Second, we approached the problem from the requirements of the intended application, and tested the material within this working range to mimic use in a prototype device and determine if the sensor would perform sufficiently. The sheath composite was optimized taking into account the polymer choice based on the mechanical properties, conductive additive (carbon black) loading to achieve the best piezoresistive performance, and conditioning to stabilize the sensor's signal. From the carbon black loading, the best performance was achieved with a high loading of $50 \mathrm{wt} \%$, which was approximately $30 \mathrm{wt} \%$ higher than the percolation threshold. The sensor's core material was varied to understand how the mechanical properties affect the sheath's piezoresistive performance, and conditioning of the sensor was completed to stabilize the sensor's signal. We tested the sensor using a random wave pattern over a 4-h period to mimic what a prototype device would be exposed to during use. We highlight the performance of the sensor for tracking strain using the piezoresistive signal by comparing root mean squared error calculations, and further improve these results by applying a machine learning algorithm to overcome any error in tracking that cannot be improved upon because of the limitations of the sensor to track efficiently in certain situations. We further explored the feasibility of using the optimized fiber strain sensor by developing a smart garment for measuring kinematic angles of the trunk relative to the pelvis in an exhaustive set of simple uniaxial and complex multiaxial movements. The design and development of this smart garment is described and, furthermore, the application of a machine learning algorithm to sensor signals for angle estimation is demonstrated. We evaluated the performance of this algorithm in a study with 12 participants performing simple to complex movements that are likely to occur in real application scenarios.

\section{Materials and Methods}

\subsection{General Materials and Methods}

Thermoplastic elastomer Hytrel 3078 (H3078) was obtained from DuPont (Kingston, ON, Canada). Polyether-urethane urea Dorlastane ${ }^{\mathrm{TM}}$ (PEU, $200 \mu \mathrm{m}$ diameter multifilament) was bought from flaxerella.com. Carbon Black Vulcan XC72R (Cabot Corporation) was purchased from www.fuelcellstore.com. All other chemicals were obtained from Sigma-Aldrich and used as received. Tensile testing was completed on a linear stage built in-house (Figure S1). Scanning electron microscopy was completed on an Explorer 3 Desktop SEM sputter coated with $10 \mathrm{~nm}$ of iridium. Melt extrusion 
was completed using a Filabot EX2 Extrusion System. Two-point sheet conductivity testing was completed with a Fluke 115 True RMS Multimeter at 1-cm spacing.

\subsection{Percolation Threshold for H3078 and Carbon Black}

Thermoplastic Elastomer Hytrel 3078 (H3078) was first dissolved in $\mathrm{CH}_{2} \mathrm{Cl}_{2}$ at 5 weight $\%$ (wt $\%$ ), and the appropriate amount of carbon black $(0,10,15,20,30,40$, or $50 \mathrm{wt} \%$ wrt H3078) was then added and stirred for $30 \mathrm{~min}$. The solutions were then cast onto glass slides, and put into a vacuum oven at $60{ }^{\circ} \mathrm{C}$ under reduced pressure for $30 \mathrm{~min}$ before conductivity testing was completed to ensure that any residual solvent was removed.

\subsection{Sensor Production}

\subsubsection{H3078 Coated with H3078:Carbon Black (50 wt\%)}

Thermoplastic Elastomer Hytrel 3078 (H3078) was extruded into a filament with a 0.4-mm die-producing filaments of ca. $450 \mu \mathrm{m}$, at a temperature of $190{ }^{\circ} \mathrm{C}$, and the filament was allowed to spool $30 \mathrm{~cm}$ below the die. The filament was fed once through a solution of $\mathrm{H} 3078$ and carbon black (50 $\mathrm{wt} \%$ ) in dichloromethane (at a concentration of $5 \% \mathrm{w} / \mathrm{v} \mathrm{H} 3078$ ) at a rate of $3.81 \mathrm{~cm} / \mathrm{second}$, and wound onto a bobbin $1.83 \mathrm{~m}$ from the exit of the coating solution (Figure 3d). Then, the bobbin containing the filament was put into a $60^{\circ} \mathrm{C}$ vacuum oven under reduced pressure for $30 \mathrm{~min}$ to ensure that any residual solvent was removed. This produced a sensor with a sheath of $30 \mu \mathrm{m}$, as determined by scanning electron microscopy (SEM) (see Figure 3e,f).

\subsubsection{PEU Coated with H3078:Carbon Black (50 wt\%)}

Pristine PEU filament was fed once through a solution of $\mathrm{H} 3078$ and carbon black ( $50 \mathrm{wt} \%$ ) in $\mathrm{CH}_{2} \mathrm{Cl}_{2}$ (at a concentration of $5 \% \mathrm{w} / \mathrm{v} \mathrm{H} 3078$ ) at a rate of $3.81 \mathrm{~cm} / \mathrm{second}$ and wound onto a bobbin $1.83 \mathrm{~m}$ from the exit of the coating solution. Then, the bobbin containing the wound filament was put into a $60{ }^{\circ} \mathrm{C}$ vacuum oven under reduced pressure for $30 \mathrm{~min}$ to ensure that any remaining solvent was removed. This produced a sensor with a sheath of $30 \mu \mathrm{m}$, as determined by scanning electron microscopy (SEM) (see Figure 3f).

\subsection{Linear Stage (Tensile and Electronic) Setup and Testing}

Tensile- and strain-resistance measurements were completed using a custom linear-stage with a Futek LFR400 (Futek Advanced Sensor Technology, Inc.; Irvine, CA, USA), connected to MATLAB (Figure S1). Testing was completed using either a sine, trapezoid, or random wave pattern (details below).

\subsubsection{Random Wave Pattern Testing}

The random wave testing was completed by first creating a random series of waypoints, and then fitting a spline function to those waypoints, with a set of boundaries from 5-20\% strain and a maximum strain rate of $15 \% \mathrm{~s}^{-1}$ for $1800 \mathrm{~s}$ (30 min). This was completed eight consecutive times with the same sensor to complete $4 \mathrm{~h}$.

\subsubsection{Normalized Root Mean Squared Error (NRMSE) Analysis}

Using software $\mathrm{R}$ and statistical package 'pracma', the position and resistance signals were normalized to values of $0-1$ using:

$$
\mathrm{S}(t)_{\text {normalized }}=\frac{S(t)-S_{\min }}{S_{\max }-S_{\min }}
$$


where $S(t)$ is the signal value at time $t, S_{\min }$ is the minimum value of that signal in each trial, $S_{\text {max }}$ is the maximum value of the signal, and $S(t)_{\text {normalized }}$ is the normalized value of the signal in time $t$. Then, the statistical analysis was completed to determine the normalized root mean squared error between the strain signal and resulting resistance signal. The result was multiplied by 100 to give a percent to one decimal place.

\subsubsection{Method for Random Forest Machine Learning Algorithm Analysis (RFMLA)}

To further increase the accuracy of tracking the applied strain in random wave testing, a random forest machine learning algorithm (RFMLA) was implemented and trained using seven 30-minute blocks of random testing from $5-20 \%$ at $15 \% \mathrm{~s}^{-1}$. Random forest is an ensemble of decision trees each trained on a bootstrap sample of training data. Bootstrap samples are generated by randomly selecting data points from training data with replacement (i.e., one data point might be selected several times) [59]. Then, a decision tree is built using each bootstrap sample, and a subset of random features is used for splitting the nodes of trees. In comparison with a single decision tree, RFMLA is robust to overfitting on the training data and has a smaller generalization error [59]. Compared with other machine learning methods such as neural network and support vector regressor, RFMLA has shown better performance for processing strain sensor data in both regression and classification tasks [60-62]. The random forest model built-in Scikit-learn [63] package of Python was used in this study, and hyper-parameters were selected to achieve the highest accuracy. The number of trees was set to 100, and the maximum number of features used for splitting nodes was set to the root square of number of features. All other parameters were selected according to the default setting.

Using the sensor's raw resistance as the input and the strain as the output of the model, a RFMLA model was trained, and its performance was evaluated by the 10 -fold cross-validation method. Raw sensor data without any normalization was used as the input to the model, hypothesizing that RFMLA would find the correlation between the raw values of resistance and strain with better accuracy than the normalized RMSE analysis, and no pre-normalization is required when using this machine learning algorithm.

\subsection{Method for Real Application Strain Range Calculation}

A motion capture system (Vicon, Oxford, UK) was used to record the position of markers on the body. Two markers were placed on the pelvis, and one marker was placed on the C7 vertebrae of a volunteer. Since the data collection was only for forward bending, using the three-marker angle of trunk relative to pelvis in the sagittal plane was calculated as the bending angle. Two other markers were placed at each end of the strain sensor stitched onto a piece of fabric, which was then attached to a stretchable undershirt (Figure 1a). The strain of the sensors was determined at two positions: (1) by standing straight—defined as pre-strain—and (2) forward bending (i.e., maximum strain, Figure 1b-d). Different locations on the back were tested to determine the best location for sensing. These motion capture markers were used to calculate strain of the sensor, which determined our working region of the sensor. 
a)

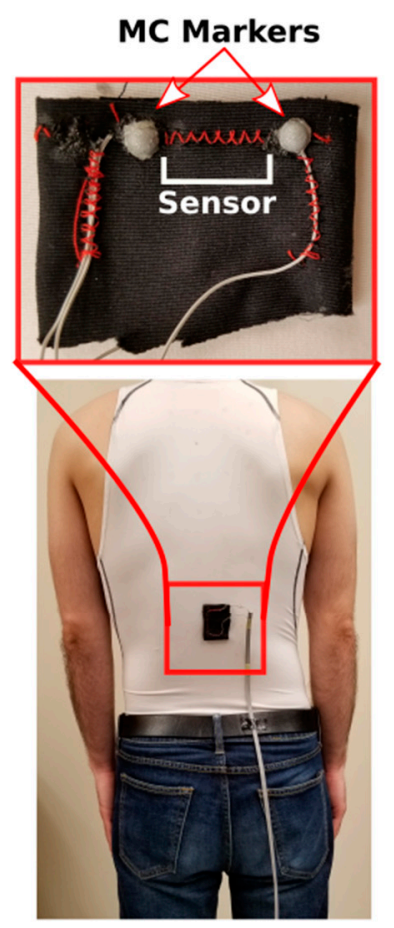

b)

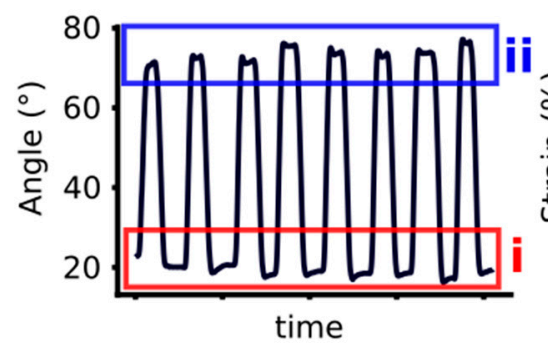

d)

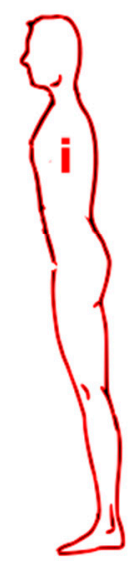

c)

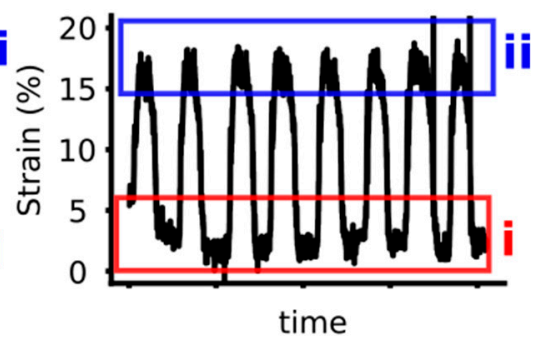

Figure 1. (a) A sample patch with motion capture markers fixed to an undershirt and worn to obtain the following values; (b) Angles obtained from a standing static position (i) to forward bending (ii); (c) Strain that is observed when going from a static standing position (i) to forward bending (ii); (d) static standing position (i) and forward bending (ii) completed for (b) and (c).

\subsection{Smart Sensor Integrated Sleeveless Shirt}

A sensor-integrated sleeveless shirt was developed to measure the three-dimensional angles of trunk movements relative to the pelvis. Our prototype was designed to be capable of measuring the trunk kinematics data in a variety of movements, including the uniaxial and multiaxial movements of the trunk. The following sections describe the elements of our design and further evaluate the prototype.

\subsubsection{Sensor Placement}

The working principle of our smart garment was to monitor fabric strain that occurs because of the user's motion. Mattmann et al. developed optical-based strain patterns of fabric on the trunk back side area while performing 27 distinct movements using a grid of reflective markers attached to the garment $[64,65]$. Within the patterns, there was consistent vertical strain along the length of the spine during trunk flexion-extension and lateral bending. Consmüller et al. used two strain gauge sensors strips attached to human skin on both sides of the spine, and using the information of two strips, showed that the upper body motions in different anatomical planes were distinguishable [66]. Consequently, we integrated two strips of sensors, each consisting of five 6-cm H3078 coated with H3078 strain sensors on both sides of the spine, covering the lower and upper back in a garment (Figure 2). Each sensor strip was $6 \mathrm{~cm}$ apart from the spine. In addition to the information that each one of these two sensor strips provided for detecting trunk flexion in forward bending, they can also be used for detecting movements in two other planes (lateral bending and rotation) by calculating the difference of the signals between the symmetrical sensors in the strips on either side of the spine. Using the strain patterns by Mattmann et al. as a starting point and conducting further empirical sensor placement tests, we found the placements shown in Figure 2 to provide the best strain patterns for trunk twisting and lateral bending $[64,65]$. This placement resulted in more sensitivity to rotation and lateral bending movements (as a result of more elongation of the sensors), which were isolated 
from the trunk flexion movement (no elongation of the sensors). In total, 18 sensors were integrated into the shirt for detecting all types of movements.
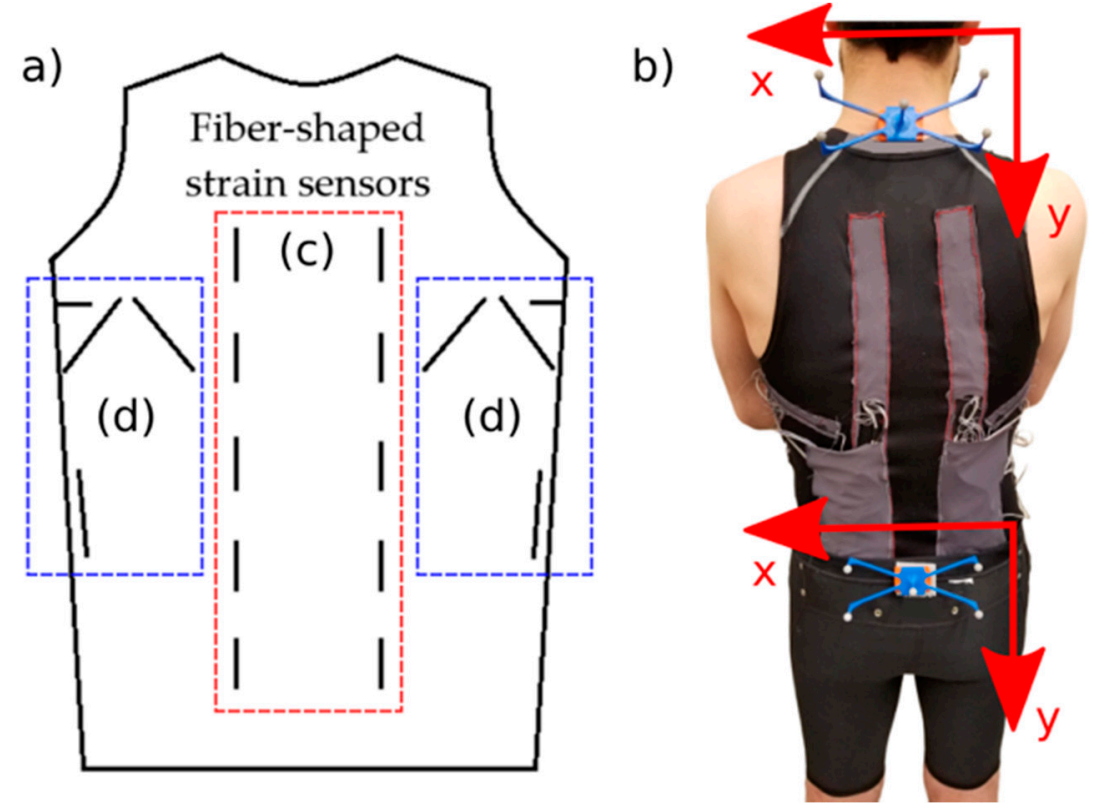

Figure 2. (a) Schematic illustration of 18 strain sensors placement on the garment. Each short line shows one strain sensor. Two vertical strips of sensors in the box (c) provide information for flexion, rotation, and lateral bending movements, whereas sensors in boxes (d) are specifically for detecting rotation and lateral bending, respectively. These sensors are isolated from trunk flexion movement. (b) The two-piece smart prototype with sensor integrated sleeveless shirt and shorts. Sensors were integrated on the back side of the shirt into the textile. The shirt was anchored to shorts with Velcro patches to minimize the upward shift at the waistline; two reflective marker sets were attached to the participant's C7 and S1 vertebras for generating reference kinematic angles using the motion capture system.

\subsubsection{Smart Sleeveless Shirt:}

The sensor's 450- $\mu \mathrm{m}$ diameter could allow the integration into textiles by stitching or weaving. This provided the potential to fabricate smart clothing with the sensors integrated directly into the textile structure. In our prototype, we attached the sensor to fabric using stitches over the length of the sensor (Figure 2). Wires were connected to the ends of the sensor using conductive ink and rubber glue. We used a commercially available tight-fitting sleeveless shirt for integrating the sensors. Eighteen sensors were integrated into the back side of the shirt to measure the strain pattern of the fabric. Shoulder and arm movements cause a significant unwanted strain pattern change in the fabric of sleeved shirts $[37,64,65]$. We intentionally chose a sleeveless shirt to reduce this unwanted change and isolate the strain pattern associated to trunk motions.

One crucial challenge of using strain-based clothing for trunk motion detection is the upward shifting of the garment at the waistline during movement. It has been shown previously that a 3-cm shift decreases the accuracy by more than $20 \%$ in an upper-body task classification problem using a smart catsuit [64,65], and care should be taken to minimize any waistline shift. The use of a leotard [67], catsuit [65], or straps [57,68] has been used to minimize this slippage. In an attempt to anchor the shirt to minimize this slippage, we opted to use a two-piece prototype (shirts and shorts) that allowed anchoring of the shirt to the shorts with Velcro patches. This allowed the comfortable extended use of this system under normal clothing without the use of a leotard/catsuit with minimal slippage. Figure 2 shows the two-piece prototype. 


\subsubsection{Experimental Setup}

A voltage divider was used for reading the change of resistance in sensors. Each sensor was connected to a resistor to form a voltage divider with a $5 \mathrm{~V}$ voltage source. The resistor value was selected to match the base resistance of the sensor, ca. $10 \mathrm{k} \Omega$. Two data acquisition boards (Models NI BNC-2110 and NI BNC-2111, National Instruments, Austin, TX, USA) were used for reading the voltage signals of all the sensors. MATLAB R2017b (The MathWorks, Inc., Natick, MA, USA) was used for data collection.

For collecting the trunk kinematics data, we used a Vicon motion capture system (Vicon, Oxford, UK). This system consisted of six infrared motion tracking cameras. Two sets of reflective markers, each with five markers (8-mm diameter) were used to track objects. These two marker sets were mounted on the participants' spinal C7 and S1 vertebras. The markers' Cartesian coordinates information was used to generate the movements' kinematic data. Figure $2 \mathrm{~b}$ shows the tracker markers attached to $a$ participant's back. A synchronization signal from the motion tracking system was used for syncing the sensors and motion capture data. Data from all the components were recorded at a frequency of $100 \mathrm{~Hz}$.

\subsubsection{Participants}

To evaluate the performance of the prototype, 12 healthy male individuals were recruited between the ages of 25-35. The experimental protocol was approved by the Office of Research Ethics at Simon Fraser University. Prior to any data collection, written informed consent was obtained from all participants. Table 1 provides the characteristics of the participants.

Table 1. Participants characteristics.

\begin{tabular}{cc}
\hline Categories & Participant \\
\hline Age (years) & $28(3.3)$ \\
Height $(\mathrm{cm})$ & $177(7.6)$ \\
Weight $(\mathrm{kg})$ & $75(9.8)$ \\
\hline
\end{tabular}

\subsubsection{Study Protocol}

The data collection for each participant lasted for $1.25 \mathrm{~h}$, including the preparation time. The shirt was anchored to the short at the waist level using three Velcro patches. The positioning of the short was adjusted based on the participant's feedback to ensure that the participants were comfortable during data collection. Then, the participants were asked to stand upright on a flat floor with feet shoulder-width apart and arms hanging to the sides of their body for the duration of the experiment. Then, a 10-s trial was recorded in this static posture. This trial was used for the standardization of all sensor data in the next trials.

The study protocol included four conditions of uniaxial movements and four conditions of multiaxial movements. Table 2 provides a list of the movements' conditions. Since people's common everyday movements include both uniaxial and multiaxial movements, an exhaustive combination of the uniaxial movements was selected as part of the study protocol. For each movement condition, participants were asked to perform the movement in three separate trials, each of them with the self-selected speeds of slow, moderate, and fast. In each trial, the movement was repeated 10 times. The range of motion of each repetition was self-selected by the participant, with a limit of maximum comfortable angle. This resulted in movements with different ranges of motion within each trial. For the random combination movement condition, participants were asked to perform random combinations of all the movements in a random order, with random ranges of motion for $60 \mathrm{~s}$. During all the trials, the participants were asked to perform the movements with a natural manner and keep the self-selected speed constant. 
Table 2. Study protocol movement conditions and types.

\begin{tabular}{ccc}
\hline Trial Number & Movement Condition & Movement Type \\
\hline $1-3$ & Rotation & Uniaxial \\
$4-6$ & Lateral Bending (LB) & Uniaxial \\
$7-9$ & Flexion & Uniaxial \\
$10-12$ & Slouching & Uniaxial \\
$13-15$ & Flexion + Lateral Bending & Multiaxial \\
$16-18$ & Flexion + Rotation & Multiaxial \\
$19-21$ & Lateral Bending + Rotation & Multiaxial \\
$22-24$ & Random Combination & Multiaxial \\
\hline
\end{tabular}

\subsubsection{Reference Angle Measurement}

Trunk reference kinematics angles (Eulerian roll, pitch, and yaw angles) were calculated using the motion capture (MoCap) data. Two marker sets were attached to the C7 and S1 vertebras. MoCap provides the Cartesian coordinates of the markers in each marker set. In each marker set, we constructed local unit vectors $X$ and $Y$ using the coordinates data and unit vector $Z$ using the cross-product of $X$ and $Y$ unit vectors (Figure $2 b$ ). Then, the relative rotation matrix between $C 7$ and $S 1$ was computed:

$$
{ }_{S_{1}}^{C_{7}} R=\left[\begin{array}{lll}
X_{C_{7}} \cdot X_{S_{1}} & X_{C_{7}} \cdot Y_{S_{1}} & X_{C_{7}} \cdot Z_{S_{1}} \\
Y_{C_{7}} \cdot X_{S_{1}} & Y_{C_{7}} \cdot Y_{S_{1}} & Y_{C_{7}} \cdot Z_{S_{1}} \\
Z_{C_{7}} \cdot X_{S_{1}} & Z_{C_{7}} \cdot Y_{S_{1}} & Z_{C_{7}} \cdot Z_{S_{1}}
\end{array}\right],
$$

in which ${ }_{S_{1}}^{C_{7}} R$ denotes the relative rotation matrix, and $X_{C_{7}}, Y_{C_{7}}, Z_{C_{7}}$ and $X_{S_{1}}, Y_{S_{1}}, Z_{S_{1}}$ are the $C_{7}$ and $S_{1}$ coordinate frame unit vectors, respectively. Each cell of this matrix was the inner product of the two vectors. Using this rotation matrix and the Z-Y-X Euler angles convention, Euler angles $\psi$ (roll, flexion angle), $\theta$ (pitch, rotation angle) and $\phi$ (yaw, lateral bending) around the lateral-medial, superior-inferior, and anterior-posterior axes were calculated. The relative orientation of the trunk (C7) with respect to the pelvis (S1) was expressed using these three angles.

\subsubsection{Signal Processing}

The voltage signals collected from strain sensors had a high-frequency noise. To remove this noise, we applied a $10^{\text {th }}$ order median filter to the voltage signal of each sensor. It has been reported that a $20-\mathrm{Hz}$ frequency was a suitable data collection frequency for monitoring normal human activities [69]; consequently, the data voltage signals were then resampled to $20 \mathrm{~Hz}$.

Slight differences between the sensors' base resistance resulted in differences between the working voltage range of the sensors. Using the signal collected in the initial static trial, we normalized each sensor signal using:

$$
S_{i}(t)_{\text {normalized }}=\frac{S_{i}(t)-S_{i, \text { mean }}}{S_{i, \text { max }}},
$$

where $S_{i}(t)$ is the sensor $i$ voltage signal at time $t, S_{i, m e a n}$ is the mean value of that sensor voltage in the static trial, $S_{i, \max }$ is the maximum value of the signal, and $S_{i}(t)_{\text {normalized }}$ is the normalized value of the signal in time $t$. Normalization brought all the signals from different sensors in the same working range.

As the placement of the sensors on the back side of the shirt was symmetrical, the difference between the signals of each symmetrical pair of sensors placed on the left and right sides of the shirt back was added to the raw data. $\left(v_{l}-v_{r}\right)$ shows this difference, in which $v_{l}$ and $v_{r}$ are the left sensor signal and the corresponding symmetrical right sensor signal, respectively. In addition to this difference, we added the derivative of the signals to the raw data set. Adding these new signals provided the angle measurement algorithm with more information for detecting multiaxial movements and improved the angle estimation accuracy. 
To generate the input for the angle measurement algorithm, a 1-s sliding window was used over the raw data signals. The window length was determined empirically by performing a grid search over windows with a length of $100 \mathrm{~ms}$ to $3 \mathrm{~s}$ to find the best performance in estimating the angles. Then, a feature extraction approach was applied to the raw signal data using the 1-s sliding window. The extracted features consisted of the minimum, maximum, mean, variance, median, root mean square, sum of absolute value, mean absolute deviation, wavelength, and slope sign changes. Then, the extracted features along with the raw data of the window were used as inputs for the angle measurement algorithm.

A random forest regressor algorithm was used for the angle estimation algorithm to train and test the model. Raw data along with the extracted features formed the input, and the roll, pitch, and yaw angles formed the output of the random forest regressor. The number of trees was set to 100, and the maximum number of features used for splitting nodes was set to the root square of number of features. All the other parameters were selected according to the default setting.

\subsubsection{Evaluation}

The performance of the angle measurement machine learning algorithm was assessed by comparing the predicted angle from the algorithm with the reference true angle measured from the motion capture system. The coefficient of determination $\left(R^{2}\right)$, the root mean squared error (RMSE), and the normalized root mean squared error (NRMSE) were used as the criteria for this comparison.

Using these criteria, we validated the performance of the machine learning algorithm in an intra-participant analysis. In this evaluation approach, one separate model was trained and tested for each participant. A three-fold cross-validation method was used to evaluate the performance of the model. Each fold comprised all the movement conditions with the same speed. Consequently, we had three folds corresponding to the slow, moderate, and fast movements. In this three-fold cross-validation approach, the model was trained using the data from two folds and tested on the remaining fold. This was repeated until all the three folds were selected as the test set. The accuracy of the model was determined by averaging the results of all three folds.

\section{Results}

\subsection{Sensor Characterization}

\subsubsection{Determining the Working Range of Desired Sensor}

The maximum strain and pre-strain that the fiber sensor would be exposed to during use was analyzed using a motion capture system. This analysis determined that the sensor was strained approximately $5 \%$ when the garment was worn without any movement, which we have defined as pre-strain (Figure 1b,d). The portion of the lower back was identified that resulted in a maximum strain of approximately $20 \%$ strain. This correlated to angles of $20^{\circ}$ for standing upright and $80^{\circ}$ for forward bending, therefore resulting in a $60^{\circ}$ range to track (Figure $1 \mathrm{~b}, \mathrm{~d}$ ). We defined the working range to be less than $30 \%$ strain for determining our sensor's basic performance below, with the random wave pattern testing strain from $5-20 \%$ to mimic a sensor in a prototype device.

\subsubsection{Sensor Fabrication, Material Selection, and Basic Sensor Properties}

The mechanical properties of the filaments were first compared with stress-strain curves, and a cyclic hysteresis test in 10\% increments up to $30 \%$ strain (minimal pre-load, $\leq 0.1 \mathrm{~N}$ ). For comparison, a commercially available conductive thermoplastic elastomer, RTP-2800, was tested analogously. RTP-2800 showed large hysteresis, increasing with strain. RTP-2800 and H3078 had hysteresis values of $55 \%$ and $7 \%$ hysteresis at $10 \%$ strain; $61 \%$ and $8 \%$ at $20 \%$ strain; and $62 \%$ and $12 \%$ at $30 \%$ strain, respectively (Figure 3a) (Hysteresis Was Calculated by Analyzing the Area under the Curve and 
Completing a Percent Difference of That Area). Polyether urethane-urea (PEU, i.e., elastane/spandex) showed no hysteresis below 30\% strain (Figure 3a).

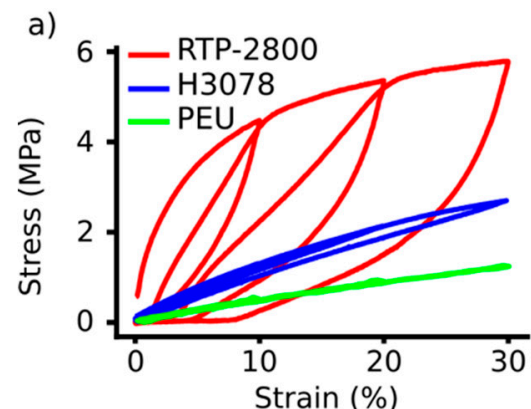

d)

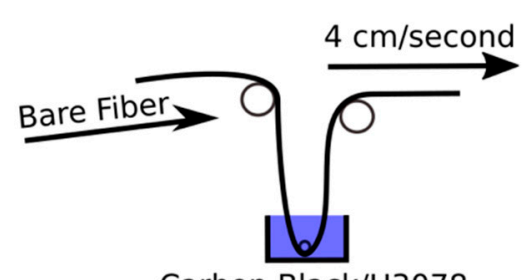

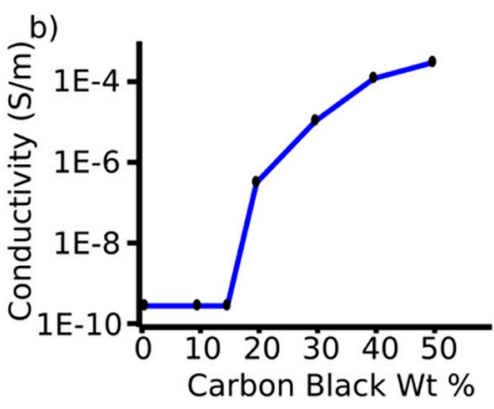

e)

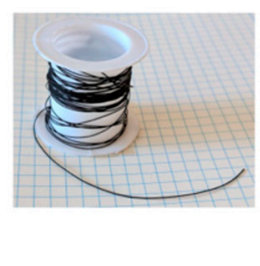

f)

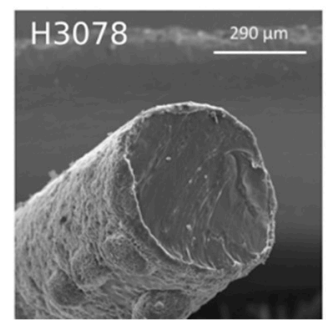

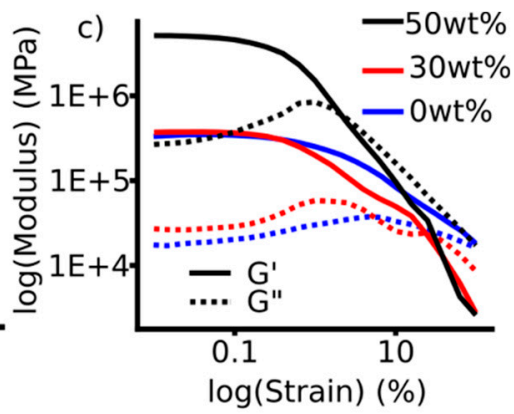

$\log ($ Strain) $(\%)$

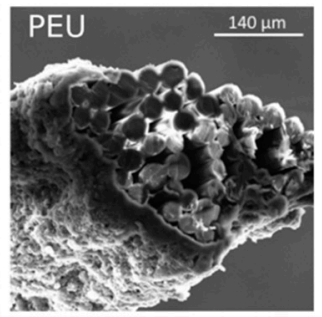

Figure 3. (a) Stress-strain curves for RTP-2800, H3078, and PEU; (b) Percolation threshold for carbon black in H3078; (c) Rheological amplitude sweep for carbon black loading in H3078; (d) Schematic for the dip-coating process of H3078 and PEU cores; (e) Resulting conductive fiber (H3078 core shown); (f) SEM image of core-sheath H3078 and PEU core sensors.

The percolation threshold — which we have defined and empirically determined as the introduction of surface conductivity and piezoresistive properties on the sheath-was found to be 20 weight $\%$ carbon black in H3078 (Figure 3b). The effect of carbon black loading in H3078 on the mechanical properties was analyzed using rheological measurements. An amplitude sweep was completed at room temperature to determine the linear viscoelastic region, elastic $\left(G^{\prime}\right)$ and storage ( $\left.G^{\prime \prime}\right)$ modulus, and the effect of carbon black addition (Figure 3c). H3078 had a storage modulus of $0.33 \mathrm{MPa}$, and a loss modulus of $0.03 \mathrm{MPa}$. With the addition of carbon black at $30 \mathrm{wt} \%$, the storage modulus and loss modulus only slightly increased to $0.38 \mathrm{MPa}$ and $0.03 \mathrm{MPa}$, respectively. The addition to $50 \mathrm{wt} \%$ carbon black significantly increased the storage and loss modulus to $5.15 \mathrm{MPa}$ and $0.27 \mathrm{MPa}$, respectively. The linear viscoelastic region with respect to carbon black loading, and the rheological measurements indicated that the addition of carbon black decreased the viscoelastic region and stiffened the material substantially in comparison to the base polymer.

The sensors were initially analyzed by completing strain-resistance measurements to determine the signal quality and accuracy within our desired working range ( $<30 \%$ strain). Sheaths produced from 30 weight $\%$ carbon black resulted in signals that suffered from hysteresis, drift, and did not track strain accurately (Figure S2). Increasing carbon black content to $50 \mathrm{wt} \%$ improved the linear piezoresistive signal tracking (Figure S2). H3078 core sensors suffered from the first cycle hysteresis and signal drift, whereas the first cycle hysteresis of PEU core sensors was less, and had a more consistent signal over 100 cycles at 30\% strain (Figure 4c, bottom graphs). Conditioning the sensors (discussed below, labeled $\mathrm{cH} 3078$ and $\mathrm{cPEU}$ ) removed the first cycle hysteresis and improved the consistency of the signal over 100 cycles. The gauge factor of the H3078:CB sheath at 30, 40, and $50 \mathrm{wt} \%$ loading was 3,4 , and 5 for up to $30 \%$ strain, respectively (Figure S2). 

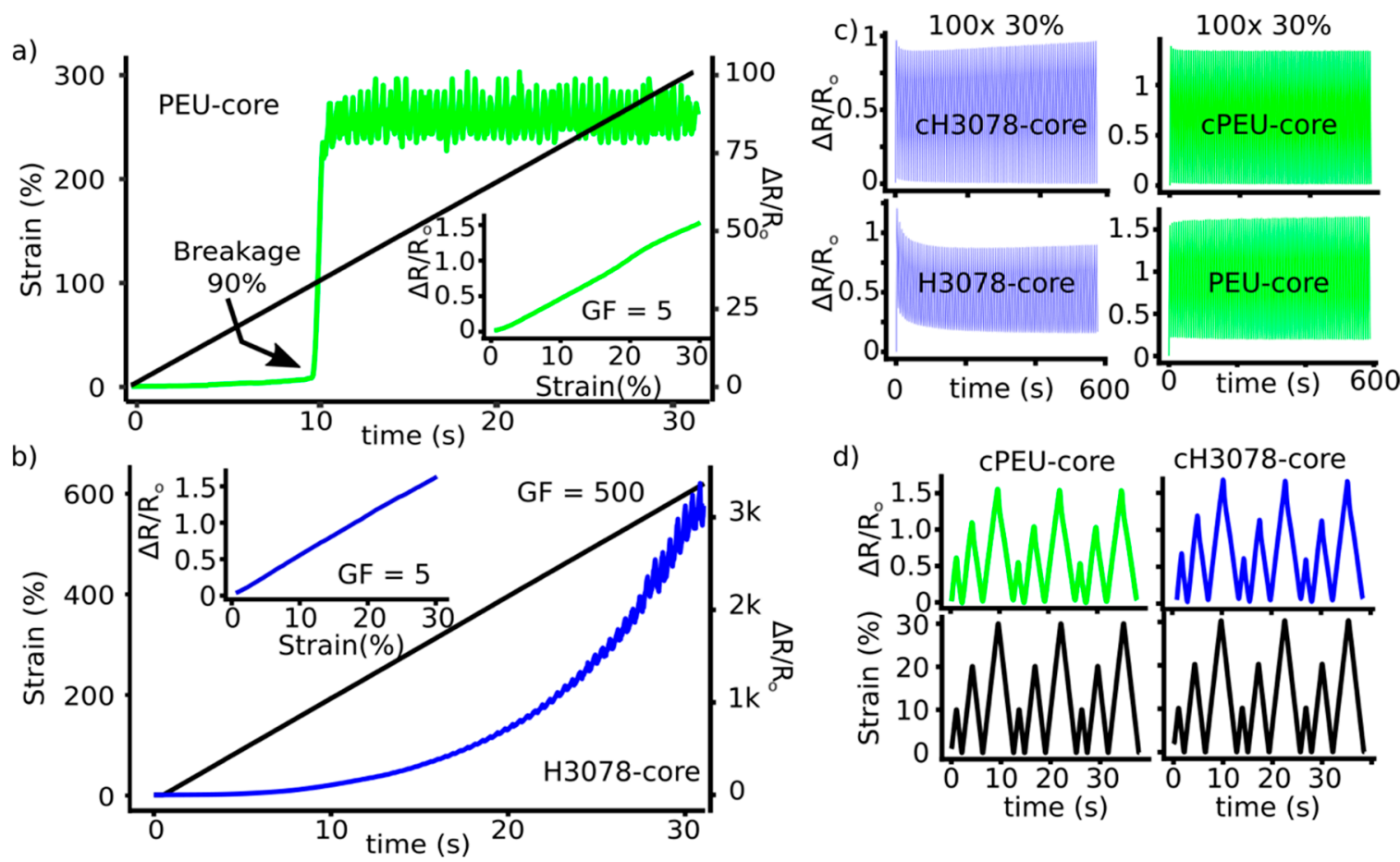

Figure 4. (a) PEU core sensor piezoresistive limit and sensor linearity up to $30 \%$ strain (inset); (b) H3078 core sensor piezoresitive limit and sensor linearity up to 30\% strain (inset); (c) 100 repetitions of 0-30\% strain for pristine $\mathrm{H} 3078$ core, PEU core, $\mathrm{cH} 3078$ core, and cPEU core sensors; (d) $10 \%, 20 \%$, and $30 \%$ trapezoid strain for three cycles with cPEU core and $\mathrm{cH} 0378$ core sensors.

\subsubsection{Strain Limit of Sensors}

The strain limit of the sensor was determined using a strain ramp experiment. A loss of piezoresistance was marked by a large increase in resistance. This data was important to identify: (1) any linear resistance-strain regions; (2) the resulting gauge factor; and (3) the strain limit of the sensing capabilities of the sensor. The H3078 core sensor was able to access large strains, upwards of $600 \%$ before a loss of piezoresistance and a maximum gauge factor of 500 (Figure $4 \mathrm{~b}$ ). The PEU core sensor was only able to undergo strains of up to $90 \%$ before a loss of piezoresistance (Figure $4 \mathrm{~b}$ ). The PEU core sensor had noticeable cracking of the H3078 carbon black sheath beyond $90 \%$ strain, which was likely a result of poor adhesion from the PEU core to the conductive H3078 carbon black sheath.

\subsubsection{Sensor Conditioning}

As previously noted, $\mathrm{H} 3078$ core and PEU core sensors underwent first cycle drift upon straining to $30 \%$ with new pristine sensors. The H3078 core sensor signal started to stabilize over 100 cycles, while the PEU core sensor was fairly consistent over 100 cycles. To overcome both the first cycle hysteresis and resistance drift, both the $\mathrm{H} 3078$ core and PEU core sensors were conditioned above the intended working range of $30 \%$ strain. This was completed at $40 \%, 80 \%$, and $120 \%$ strain for 100 cycles using a sinusoidal wave pattern at a strain rate of $10 \% \mathrm{~s}^{-1}$. Since the PEU core sensors had a limit of $80-90 \%$ strain, this was only completed at $40 \%$ for those samples. The sheaths of H3078 core sensors were imaged using scanning electron microscopy to observe any obvious damage to the sheath. The conditioning at $80 \%$ and $120 \%$ strain produced larger cracking and gaps in the conductive sheath, which were not observed in the pristine and 40\% conditioned samples (Figure S3). The $80 \%$ and 120\% conditioned samples also suffered from lower performance, and therefore, the $40 \%$ conditioning was chosen for more in-depth testing: these were labeled the cH3078 core and cPEU core sensors. The stability of the piezoresistive signal of $\mathrm{cH} 0378$ cores and cPEU cores were tested analogously to pristine 
sensors over 100 cycles from $0-30 \%$ strain, resulting in no first cycle hysteresis and a consistent signal (Figure 3c).

\subsubsection{Sensor Linearity}

Ideal piezoresistive sensors possess a stable and linear relationship between resistance and strain. This requires a consistent gauge factor within the working range. After conditioning the $\mathrm{H} 3078$ and PEU core sensors ( $\mathrm{cH} 3078$ core and cPEU core), both sensors had ideal linear relationships within the working range of $30 \%$ strain (Figure $4 \mathrm{a}$,b inset graphs). Furthermore, both sensors had little first cycle drift, and could be repetitively cycled to $10 \%, 20 \%$, and $30 \%$ strain without signal drift or a change in gauge factor (Figure $4 \mathrm{~d}$ ).

\subsubsection{Effect of Strain Rate on Sensor Performance}

The effect of strain rate on the sensors was tested by completing a simple $0-10 \%$ sinusoidal wave pattern while varying only the strain rate. This was completed at a higher frequency $(1 \mathrm{~Hz})$ and lower frequency of $(0.1 \mathrm{~Hz})$ for both $\mathrm{cH} 3078$ and cPEU core sensors (Figure S4). Both cH3078 and cPEU core sensors suffer from a small amount of rate-dependent piezoresistive properties at $1 \mathrm{~Hz}$, as indicated by the decreasing piezoresistive signal versus that of one that is consistent at the slower strain rate of 0.1 $\mathrm{Hz}$ [70-72].

\subsubsection{Sensor Testing: Random Wave Pattern to Simulate Real Events}

With both the pristine and conditioned sensors (H3078 core, PEU core, $\mathrm{cH} 3078$ core, and cPEU core, respectively) we sought a methodology that would allow us to mimic the conditions that the sensors would be exposed to in prototype devices. The methodology employed utilizing the known working range of the sensor for our specific application, which we defined (5-20\% strain) with a strain rate up to $15 \% \mathrm{~s}^{-1}$. Random wave pattern testing was completed in $30-\mathrm{min}$ intervals, for a total of $4 \mathrm{~h}$ on both non-conditioned (H3078 core and PEU core sensors) and conditioned (cH3078 core and cPEU core sensors). Empirically, the average resistance values of H3078 core sensors increased over $4 \mathrm{~h}$ (Figure 5a). The PEU core sensors gauge factor was increasing over the $4 \mathrm{~h}$, although the baseline resistance stayed consistent (Figure 5c). The cH3078 core and cPEU core sensors did not have any of the noticeable signal drift or change of baseline that was observed in non-conditioned samples (Figure 5b,d).

Analysis of these 4-h random wave pattern test experiments was completed initially by two simple methods. First, each 30-min block was normalized, and the accuracy of the sensor was determined using a normalized root mean square error (NRMSE) calculation (see the Methods section for details). The second method involved concatenating the 30-min blocks, followed by NRMSE calculations. The second of these two methods highlighted the variability of the sensor signal throughout the 30-min blocks (which was visually apparent in Figure 5a,c, as discussed above). This gave an indication of the consistency of the sensor over longer periods of testing. Pristine H3078 core sensors had an average NRMSE of $7.7 \%$ when normalized within the 30 -min testing intervals, and increased to $15 \%$ when normalized as a four-hour testing block (see Table 3 for all NRMSE values). Pristine PEU core sensors had an NRMSE of $9.3 \%$ when normalized within the 30-min testing intervals, and increased to an NRMSE of $16 \%$ when normalized as a 4-h block. The cPEU core NRMSE in the 30-min normalization calculations decreased to $5.0 \%$ from $9.3 \%$, while the $\mathrm{cH} 3078$ core sensors decreased to $7.1 \%$. Normalizing the concatenated 4-h testing block, the cPEU core sensor increased in performance to an NRMSE of $6.3 \%$ from $16 \%$, and the $\mathrm{cH} 3078$ core sensor improved to $6.4 \%$ from $15 \%$. 
a) $\mathrm{H} 3078$-core

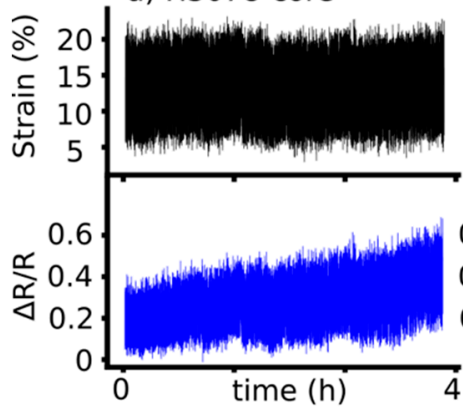

c) PEU-core

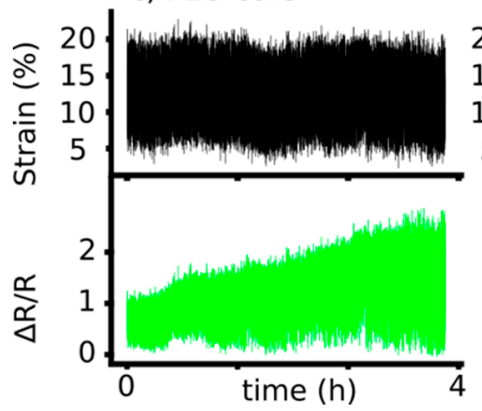

b) $\mathrm{cH} 3078$-core

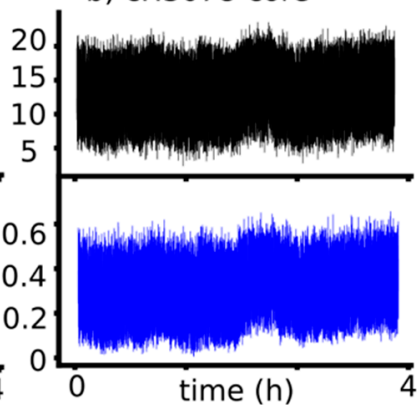

d) CPEU-core

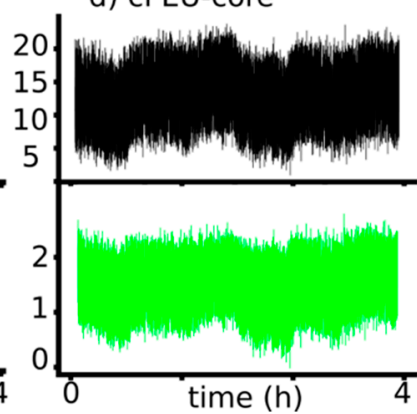

e) $\mathrm{cH3078-core} \quad$ Actual Strain

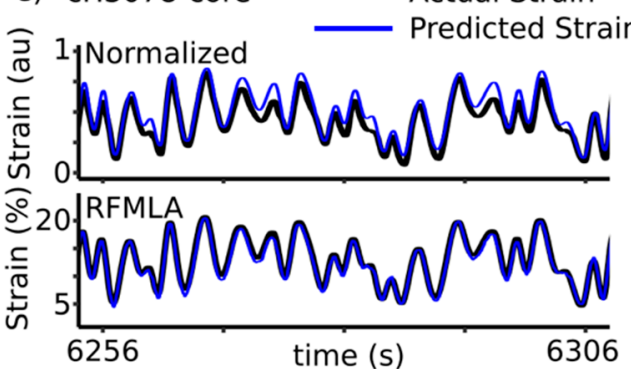

f) CPEU-core

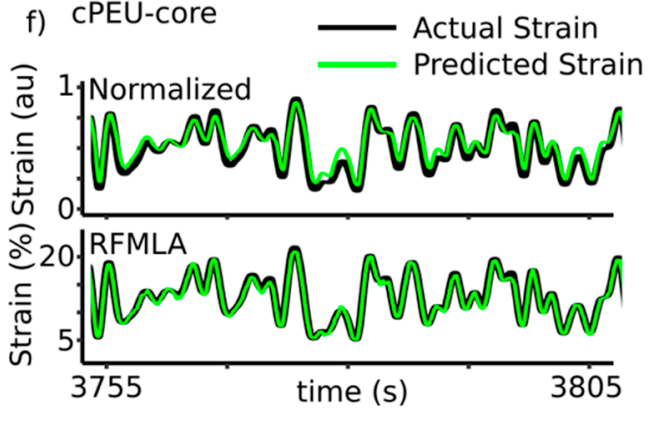

Figure 5. Random wave testing between 5-20\% strain (top, black) and resistance signal (bottom) for (a) an H3078 core sensor (blue), (b) a cH3078 core sensor (blue), (c) a PEU-core (green), and (d) a cPEU core sensor (green); (e) Snapshot overlay of predicted strain using normalized resistance signals (top) and random forest machine learning algorithm (RFMLA) predicted strain (bottom) for the random wave testing for $\mathrm{cH} 3078$ core sensors; (f) Snapshot overlay of predicted strain using normalized resistance signals (top) and RFMLA predicted strain (bottom) for the random wave testing for CPEU core sensors.

Table 3. Normalized root mean squared error (NRMSE) data for H3078 core and PEU core sensors with different analysis procedures.

\begin{tabular}{cccc}
\hline Sample & NRMSE $^{\mathbf{a}}$ & NRMSE $^{\mathbf{b}}$ & RFMLA NRMSE $^{\mathbf{c}}$ \\
\hline H3078 core & $7.7 \%$ & $15 \%$ & - \\
cH3078 core & $7.1 \%$ & $6.4 \%$ & $1.6 \%$ \\
PEU core & $9.3 \%$ & $16 \%$ & - \\
cPEU core & $5.0 \%$ & $6.3 \%$ & $2.6 \%$ \\
\hline
\end{tabular}

${ }^{\text {a }}$ Normalized each 30-min section. ${ }^{\mathrm{b}}$ Normalized over complete 4 -h testing period. ${ }^{\mathrm{c}}$ No normalization completed before RFMLA analysis.

\subsubsection{Sensor Optimization Using Machine Learning Algorithms}

With the raw data resulting in NRMSE values as low as 5\%, we sought to further increase the accuracy of the sensors' tracking of strain using the piezoresistance by training a random forest machine learning algorithm (RFMLA) using seven 30-minute blocks of random testing and analyzing a separate 30-min testing block. RFMLA has shown promising performance in the regression on strain sensor's data for our prototypes [60-62]. The use of RFMLA improved the tracking of the strain with cH3078 core sensors to a NRMSE of $1.6 \%$, while the cPEU core sensors improved to $2.6 \%$.

\subsection{Smart Sleeveless Shirt Testing}

For each participant, one-hour trunk motion data comprised of 24 different trials was collected. In each movement type, only the relevant sensors of that movement type were strained so that the voltage patterns were different between different movement types (Figure S5). Averaged across all uniaxial and multiaxial movement conditions, the random forest regressor estimated the roll angle 
with $R^{2}=0.94 \pm 0.02, \mathrm{RMSE}=4.26^{\circ} \pm 0.73^{\circ}$, and normalized $\mathrm{RMSE}(\mathrm{NRMSE})=5.14 \% \pm 0.64 \%$; the pitch angle with $R^{2}=0.92 \pm 0.02$, RMSE $=3.53^{\circ} \pm 0.52^{\circ}$, and NRMSE $=4.68 \% \pm 0.58 \%$; and the yaw angle with $R^{2}=0.91 \pm 0.03, \operatorname{RMSE}=3.44^{\circ} \pm 0.52^{\circ}$, and NRMSE $=5.79 \% \pm 0.73 \%$ averaged over all participants (Table S1 for the details of all participants). Table 4 reports the detailed performance of the algorithm for each of the three self-selected speeds. Among the three angles, movements with moderate speed had the best performance with the maximum accuracy $\left(R^{2}=0.96\right.$ in flexion angle), whereas fast movements had lower angle estimation accuracies $\left(R^{2}=0.90\right.$ in rotation angle).

Table 4. Performance results of the algorithm in the detection of three angles of $\psi$ (flexion), $\theta$ (rotation), and $\phi$ (lateral bending) for different speeds averaged across all participants. NRMSE: normalized RMSE.

\begin{tabular}{cccccccccc}
\hline & \multicolumn{3}{c}{$\boldsymbol{\Psi}$} & \multicolumn{3}{c}{$\theta$} & \multicolumn{2}{c}{$\boldsymbol{\phi}$} \\
\hline Speed & $R^{2}$ & $\begin{array}{c}\text { RMSE } \\
\mathbf{( D e g )}\end{array}$ & $\begin{array}{c}\text { NRMSE } \\
\mathbf{( \% )}\end{array}$ & $R^{2}$ & $\begin{array}{c}\text { RMSE } \\
\mathbf{( D e g )}\end{array}$ & $\begin{array}{c}\text { NRMSE } \\
\mathbf{( \% )}\end{array}$ & $R^{2}$ & $\begin{array}{c}\text { RMSE } \\
\text { (Deg) }\end{array}$ & $\begin{array}{c}\text { NRMSE } \\
(\%)\end{array}$ \\
\hline Slow & 0.94 & 4.12 & 5.05 & 0.93 & 3.35 & 4.70 & 0.92 & 3.26 & 5.73 \\
Moderate & 0.96 & 3.61 & 4.55 & 0.94 & 3.12 & 4.09 & 0.93 & 3.03 & 5.10 \\
Fast & 0.92 & 5.06 & 5.82 & 0.90 & 4.12 & 5.25 & 0.88 & 4.02 & 6.55 \\
Average & $0.94(0.02)$ & $4.26(0.73)$ & $5.14(0.64)$ & $0.92(0.02)$ & $3.53(0.52)$ & $4.68(0.58)$ & $0.91(0.03)$ & $3.44(0.52)$ & $5.79(0.73)$ \\
\hline
\end{tabular}

Table 5 presents the detailed results of angle estimation in each movement condition among all participants. The algorithm estimated the principal angles in uniaxial movements accurately $\left(R^{2}>0.97\right)$. As the movement became more complex, the error in estimating angles increased with the maximum error happening in the random combination movement condition (NRMSE between 7\%-10\%).

Table 5. Performance results of the algorithm in the detection of three angles of $\psi$ (flexion), $\theta$ (rotation), and $\phi$ (lateral bending) for each movement condition. NRMSE: normalized RMSE.

\begin{tabular}{|c|c|c|c|c|c|c|c|c|c|}
\hline \multirow[b]{2}{*}{ Movement } & \multicolumn{3}{|c|}{$\psi$} & \multicolumn{3}{|c|}{$\theta$} & \multicolumn{3}{|c|}{$\phi$} \\
\hline & $R^{2}$ & $\begin{array}{l}\text { RMSE } \\
\text { (deg) }\end{array}$ & $\begin{array}{c}\text { NRMSE } \\
(\%)\end{array}$ & $R^{2}$ & $\begin{array}{l}\text { RMSE } \\
\text { (deg) }\end{array}$ & $\begin{array}{c}\text { NRMSE } \\
(\%)\end{array}$ & $R^{2}$ & $\begin{array}{l}\text { RMSE } \\
\text { (deg) }\end{array}$ & $\begin{array}{c}\text { NRMSE } \\
(\%)\end{array}$ \\
\hline Rotation & 0.94 & 3.23 & 3.91 & 0.97 & 1.66 & 2.41 & 0.94 & 2.45 & 3.73 \\
\hline Lateral Bending & 0.93 & 3.65 & 4.32 & 0.83 & 3.60 & 3.07 & 0.98 & 1.94 & 2.84 \\
\hline Flexion & 0.97 & 3.08 & 3.49 & 0.85 & 4.16 & 3.52 & 0.87 & 3.13 & 4.34 \\
\hline Slouching & 0.95 & 2.39 & 3.39 & 0.93 & 1.17 & 2.85 & 0.94 & 2.12 & 4.26 \\
\hline Flexion + Lateral Bending & 0.87 & 4.27 & 8.78 & 0.89 & 4.08 & 4.85 & 0.92 & 3.12 & 6.04 \\
\hline Flexion + Rotation & 0.92 & 4.60 & 7.10 & 0.87 & 4.36 & 6.83 & 0.82 & 3.02 & 8.03 \\
\hline Lateral Bending + Rotation & 0.92 & 4.29 & 7.25 & 0.86 & 4.26 & 5.83 & 0.97 & 3.38 & 5.42 \\
\hline Random Combination & 0.85 & 5.13 & 7.74 & 0.83 & 4.90 & 10.67 & 0.85 & 4.48 & 10.09 \\
\hline
\end{tabular}

Figure 6 demonstrates all three angles in four multiaxial movement conditions (see Figure S6 for an example of uniaxial movement conditions). Random forest regressor estimation follows the actual angle pattern in all complex movements $\left(R^{2}>0.82\right)$. The flexion angle had the minimum decrease in accuracy with $R^{2}=0.85$ in the random combination movement condition. 


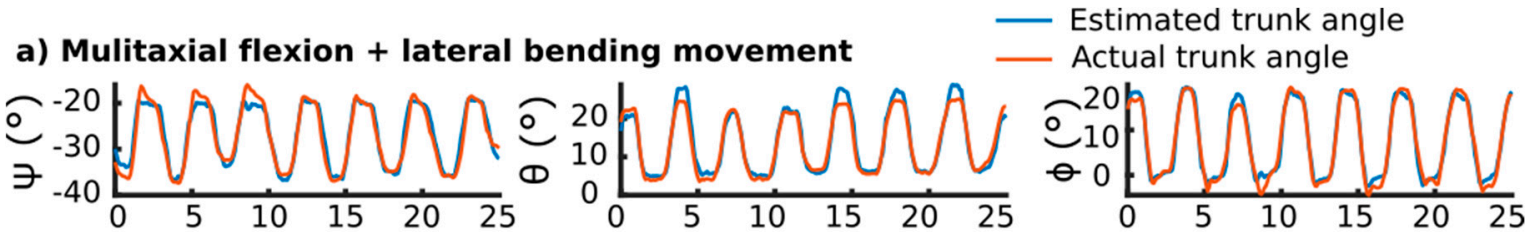

b) Multiaxial flexion + rotation movement
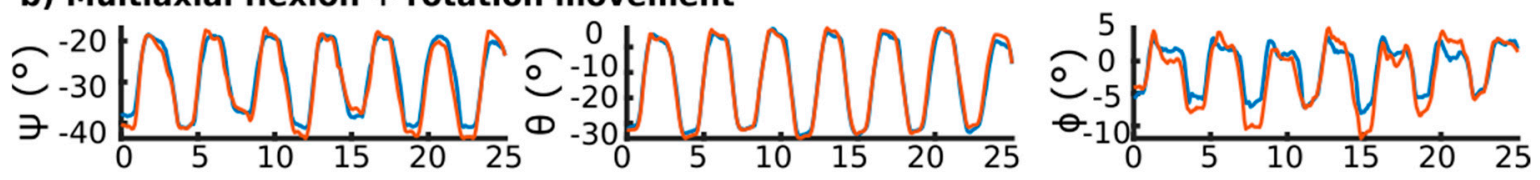

c) Multiaxial lateral bending + rotation movement
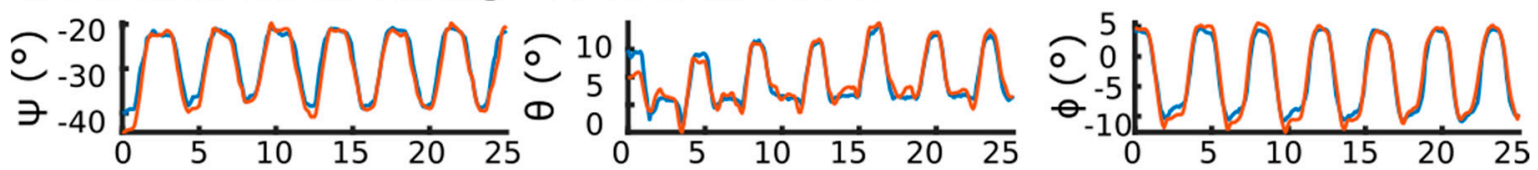

d) Multiaxial random combinational movement
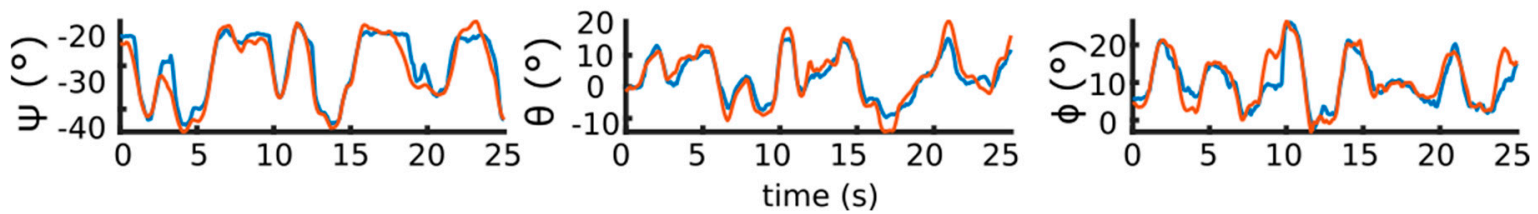

Figure 6. Exemplary comparison between three kinematic reference and estimated $\psi$ (flexion), $\theta$ (rotation), and $\phi$ (lateral bending) angles in multiaxial movements. Most estimation errors happened at peaks where the algorithm cannot estimate the exact value. (a) Flexion and lateral bending movement; (b) Flexion and rotation movement; (c) Lateral bending and rotation movement; and (d) Multiaxial random combination movement.

\section{Discussion}

We hypothesized that creating a fiber sensor with a supportive non-conductive elastic core covered with a conductive sheath would improve the common problems associated with conductive composite sensors - such as a decrease in viscoelastic region and stiffening of the polymer. Piezoresistive sensors rely on the electrical disconnections of conductive additives within the polymer matrix upon the application of strain [52]. If plastic deformation occurs, the baseline resistance values and gauge factors will change. This makes using the piezoresistive signal difficult for tracking strain accurately. A core-sheath morphology could provide the required support for the piezoresistive composites in a standalone morphology that could be truly integrated seamlessly into textile base wearable devices.

Multidisciplinary research combining both sensor design, fabrication, and optimization; subsequent use in prototype devices for use in larger cohort kinematic tracking studies has yet to be reported. Our goal for this report was to fabricate a sensor that:

(1) Could be made from readily available materials.

(2) Would be reliable and accurate within the intended working range for our application.

(3) Would be accurate at tracking strain when exposed to random movements that are typical in prototype devices over longer testing periods.

The performance of sensors has been improving dramatically with recent reports accessing strains upwards of $1000 \%$ [36,53], large gauge factors exceeding $10^{6}$ [73], and resistance-strain linearity beyond $120 \%$ strain [36]. A more in-depth larger cohort testing for the accuracy and feasibility of these sensors for three-dimensional kinematic movement has been much less common, and most sensor reports have limited proof-of-concept results. Furthermore, our aim to provide a methodology to test sensors in a way that mimics prototype devices would allow a rigorous analysis of future sensors prior to any 
larger cohort prototype device testing, resulting in better performance with more accurate kinematic data for analysis.

To begin the fabrication of the sensors, we explored a variety of conductive additives, but chose a high conductive grade of carbon black. This was simply the lowest cost conductive additive requiring no specialized mixing methods such as sonication or shear mixing when using polymer solutions. H3078, a thermoplastic elastomer with low hysteresis within the desired working range $(<30 \%$ strain, Figure 3a), required 20 weight $\%$ carbon black to reach the percolation threshold, but had the best performance at 50 weight $\%$ loading. This high loading of carbon black affects the mechanical performance of the material by increasing both the elastic and storage modulus of the material and lowering the viscoelastic region of the material (Figure 3c). This unfortunately decreased the ideal elastic properties of the material, and would result in more plastic deformation of the composite if used independently as a material for a fiber sensor. This would cause a detrimental piezoresistive baseline signal drift because of the lack of reconnections within the conductive pathways of the composite after plastic deformation. Notably, these have been used previously, but with a supporting elastic covering to reduce large plastic deformations $[37,57,58]$. We hypothesized that including an elastic core would support a piezoresistive sheath—within the core's elastic region-and decrease the amount of problematic piezoresistive signal baseline drift, since the sheath would return to its original length, and the core would be dominating the mechanical properties over the thinner sheath layer. Two core materials were chosen: A thermoplastic elastomer H3078- the same material used for the piezoresistive sheath—and PEU—otherwise known as spandex—a ubiquitous highly elastic, low hysteresis polymer.

Applying the conductive composite to the cores was completed using a one-step dip-coating process. The advantage of using a piezoresistive polymer composite sheath was the ease of application, requiring only one dip-coating step. Typically with graphene or carbon nanotube coatings, multiple coatings or sequences are required to deposit enough conductive material to create a piezoresistive layer $[36,54]$. This technique is attractive, since it could be implemented easily as a finishing step during fiber production using a roll-to-roll manufacturing process such as those used for wet spinning, wet-dry spinning, melt spinning, or electrospinning [74].

The accessible piezoresistive sensing strain of the H3078 core sensors was higher compared to the PEU core sensors. The PEU fiber was more elastic in comparison to H3078 fibers beyond $30 \%$, and could withstand large strains beyond $1000 \%$, but the adhesion between the core and sheath were such that the sheath underwent irreversible damage beyond $90 \%$ strain, causing a loss of signal. There was also noticeable cracking in the sheath of the PEU core sensor beyond $90 \%$. Some sensors that are produced from pure conductive materials such as carbon nanotube forests or Ag nanoparticles rely on cracking for piezoresistance $[56,73,75]$; however, for piezoresistive composites, this is a point of failure. It was concluded that the H3078 core fibers' advantage of using a one-step dip-coating process with a solvent that softens/dissolves a thin layer of the outer portion of the core allowed better adhesion of the sheath to the core by increasing polymer entanglement at the interface [76]. Producing PEU core sensors with a PEU-based sheath could have improved the strain limit of the sensor, although this was not explored, since PEU was only soluble in high boiling point solvents (such as $\mathrm{N}, \mathrm{N}$-dimethyl formamide or N-methyl-2-pyrrolidone) that did not permit coating with an analogous procedure.

Both the H3078 core and PEU core sensors required conditioning (stretching the sensor repetitively to a specific strain) prior to use. It was observed that $\mathrm{H} 3078$ core sensors had large first cycle hysteresis and a signal drift that stabilized over 100 cycles at 30\% strain (Figure $4 \mathrm{~d}$ ). PEU core sensors did not suffer from as large a first cycle hysteresis, and had a consistent signal throughout 100 cycles at $30 \%$ strain (Figure $4 \mathrm{~d}$ ). It was hypothesized that in H3078 core sensors, both the core and sheath were undergoing some plastic deformation in these initial tests, and the sheath was undergoing conductive pathway destruction $[73,77]$. The PEU of the PEU core sensors were not undergoing plastic deformation-which would explain the smaller first cycle hysteresis, since the sheath material would return to its original length - and the stability of the signal was likely directly related to the higher elasticity and low hysteresis of the PEU core. The sheath was able to return to its original length 
because of the elasticity of the PEU core, and therefore reconnect the majority of conductive pathways, minimizing signal drift. To eradicate any piezoresistive signal drift and first cycle hysteresis, both sensors were conditioned above the working range of $30 \%$ to strains of $40 \%, 80 \%$, and $120 \%$ for 100 repetitions. PEU core sensors were only able to undergo conditioning at $40 \%$ strain. SEM imaging of the conditioned sensors revealed larger cracking in the sheath for the $80 \%$ and $120 \%$ conditioned sensors (Figure S3). Furthermore, sensors conditioned at $80 \%$ and $120 \%$ decreased in performance, and were therefore discarded from further testing. Conditioning to $40 \%$ strain did not noticeably affect the sheath's morphology by SEM, and improved the performance such that we were able to track $10 \%$, $20 \%$, and $30 \%$ strains repetitively and accurately with a stable baseline with both cH3078 and cPEU core sensors (conditioned sensors, Figure 4f). These sensors also had a linear correlation for a change in resistance to strain up to $30 \%$ with a gauge factor of five (Figure 4e). Recent reports of core-sheath fiber strain sensors have similar gauge factors at low strain [44,54], while most have strived to achieve high gauge factors at larger strains that are not required for this type of application.

Finally, for sensor characterization, we explored the limitations of the sensor. The high strain limit of the H3078 core sensor was 600\% with a maximum gauge factor of 500, while PEU core sensors were only able to access $90 \%$ strain. Therefore, the sensors were well above the required strain to perform in our intended application. Strain rate dependence was explored using two frequencies of 0.1 and $1 \mathrm{~Hz}\left(2 \% \mathrm{~s}^{-1}\right.$ and $20 \% \mathrm{~s}^{-1}$, respectively). The piezoresistive signal underwent a small amount of rate-dependent hysteresis and signal compression at the higher 1-Hz strain rate (Figure S4) [71-73]. The slower strain rate of $0.1 \mathrm{~Hz}$ did not show this phenomenon. Piezoresistive sensors have been reported that are able to track at higher strain rates, although they do not utilize polymer composites and rely on crack formation and decreased contact as the mechanism of piezoresistivity, avoiding this effect from the polymer mechanical properties [78]. If fast strain rates are required, the use of capacitive sensors are more appropriate, since they do not depend on the piezoresistive mechanism susceptible to rate-dependent hysteresis, and can sense accurately at strain rates upwards of $50 \% \mathrm{~s}^{-1}$ [79]. This limitation of the sensor is directly related to the inherent material properties, yet for signal analysis, this information is useful. These limitations may indicate the type of movements and scenarios in prototype testing that result in lower accuracy. Furthermore, if the rate-dependent signal effects are predictable, analysis using machine learning algorithms may be capable of predicting the actual strain profile.

With cH3078 core and cPEU core sensors in hand, we created a testing protocol that would allow us to monitor both tracking accuracy within our defined working region $(<30 \%$, specifically $5-20 \%)$ and the effect of random movements that are likely to occur during a larger cohort prototype testing of a wearable device with these sensors. We sought a testing protocol that would mimic prototype testing conditions to first understand how the sensors behave in different conditions, and how accurate we could get to predicting the strain of the sensors when exposed to random motion that would be seen in prototype devices. Using the results of this testing protocol, we further used the optimized $\mathrm{cH} 3078$ core sensor to develop a smart textile garment that is capable of tracking three-dimensional random back movements, and evaluated the performance of the sensor in a prototype device for tracking movements that are most likely to happen in real applications of the sensor. Thus far, this type of testing to mimic a prototype device to gauge performance has not been reported.

The 4-hour testing blocks were completed to mimic the constant change of strain and strain rate that prototype devices would be expected to track over a day of use. We tracked H3078, PEU, cH3078, and cPEU core sensors to further show the benefit of conditioning the sensors prior to use (Figure $5 \mathrm{a}-\mathrm{d}$ ). With conditioning, both the $\mathrm{cH} 3078$ and cPEU core sensors had a stable resistance signal over the 4-h testing periods, which was highlighted by the sharp drop in NRMSE when calculated by concatenating the signals over the full 4-h with NRMSEs at $6.4 \%$ and $6.3 \%$, respectively (Figure $5 c, d$, Table 3 ). These results are unique, since we obtained a performance metric from a test that is likely more demanding than what a normal person would expose these sensors to, with accuracies similar to those of other wearable technologies [80]. 
While this result was promising, we have previously reported the use of machine learning to increase accuracy in sensor tracking after normalization [60]. We hypothesized that using machine learning algorithms could improve the tracking if the events causing tracking errors were reproducible and consistent during the random wave pattern testing. Reducing the amount of signal processing would also be advantageous in prototype devices; therefore, we tested the accuracy of RFMLA without any signal normalization. Raw signals analyzed by the RFMLA resulted in the lowest NRMSE values of $1.6 \%$ and $2.6 \%$ for $\mathrm{cH} 3078$ and cPEU core sensors. This indicated that the sensors were in fact responding reproducibly and consistently throughout our 4-h testing period, and the RFMLA was able to adjust the predicted strain to higher accuracy. These results indicate that this sensor would be capable of measuring the angle of the lower back in complicated multiplanar movements. However, careful design of prototypes considering optimized sensor placement, modified signal preprocessing, and specific data analysis approaches are still required to achieve promising results in tracking the angles of three-dimensional movements.

Developing wearable motion capture systems for unobtrusive daily use is an ongoing challenge. In this work, we developed a smart sleeveless shirt by integrating the $\mathrm{cH} 3078$ core strain sensors into a commercially available garment. We investigated the feasibility of this smart garment in capturing all types of planar uniaxial and complicated multiaxial trunk motions.

The results of our work showed a high agreement between the estimated angles using the smart garment and the angles calculated from the motion capture system. All three angles were estimated with an RMSE of less than $4.26^{\circ}$ averaged over all movement conditions. Cuesta et al. suggested that an error between $2-5^{\circ}$ is likely to be regarded as an acceptable accuracy for wearable systems, while extra consideration is required for clinical applications [80]. The accuracy of our system fell within this range, and would be sufficient for a wearable motion tracking system. Furthermore, there was a high correlation between the estimated angle and real angle, with an $R^{2}$ higher than 0.91 for all angles. Similar to other systems, the correlation of estimate to real angles was affected by the movement complexity [81]. Our results showed that in simple planar movements, the estimation followed the real pattern accurately $\left(R^{2}>0.97\right)$. As the movement became more complex, the level of agreement between the estimated and real angles decreased $\left(R^{2}>0.83\right)$, but the RMSE error stayed below $5^{\circ}$.

The random forest regressor algorithm estimated the trunk flexion, rotation, and lateral bending with RMSEs of $4.26^{\circ}, 3.53^{\circ}$, and $3.44^{\circ}$, respectively in our system. IMU-based motion captures are the most common wearable motion captures. Several previous studies have investigated the validity of IMU-based systems for motion analysis [26,27]. Schall et al. reported an accuracy of $4.1^{\circ}$ to $6.6^{\circ}$ for trunk motion monitoring during a field-based study [82]. Samadani et al. reported errors less than $2^{\circ}$ for trunk planar angle measurement [30]. Our results compare well to the field-based study, but they fall lower than the results of Samadani et al. It should be emphasized that the data collection protocol in the Samadani et al. report was simple, including only five repetitions of planar movements, while in other studies with more complicated protocol, an error between $2-5^{\circ}$ was reported for these IMU-based systems [80]. Considering IMUs' limitations of susceptibility to magnetic disturbances and drifting over time $[83,84]$, our developed smart shirt is comparable to IMU-based systems and superior for environments where magnetic distortions are present. This is the case for most environments in which ferromagnetic materials such as metals are found.

To the best of our knowledge, there have been limited studies investigating the feasibility of smart garments based on textile-based sensors measuring angles during multiaxial trunk motions. Mokhlespour et al. developed a smart undershirt that was capable of measuring planar lumbar movements with $1.3^{\circ}$ error when measuring single degree-of-freedom movements [57]. Therefore, no conclusions could be drawn about the application of their system to daily usage where multiaxial movements are inevitable. Mattmann et al. developed a smart catsuit using similar sensors for task classification, although no angle measurement was completed [64,65]. Yamamoto et al. used stretch sensors fixed to the skin for measuring complicated lumbar motion angles [58]. Complicated movements resulted in errors higher than $10^{\circ}$ in measuring trunk flexion-extension angles. Our 
study showed superior results in measuring trunk three-dimensional angles while performing complicated movements.

Among the three estimated kinematic angles, the pitch angle (corresponding to trunk rotation) was estimated with $4.68 \%$ error. It has been previously shown that the most challenging angle to measure is the trunk rotation angle [85]. Measuring trunk rotation angles using IMU-based systems has been difficult because of the susceptibility of IMU sensors to magnetic distortion $[31,83]$, which is a problem where textile-based sensors could provide an alternative, accurate solution.

The accuracy of the prototype device did have dependence on the speed of movements, although there was only minimal increase in error during fast movements by only $1.5^{\circ}$ for flexion, and $1^{\circ}$ for rotation and lateral bending.

Overall, our developed smart tank top system was able to track multiaxial trunk movements in three dimensions with an error less than $4.26^{\circ}$. This shows the great potential of smart textile systems to be used as wearable motion tracking systems. This will provide us with tools for long-term unobtrusive data collection from human movements. This data could supply useful information for applications such as developing personalized interventions to decrease the occurrence of lower back pain among health care workers.

\section{Conclusions}

A core-sheath piezoresistive sensor was produced from readily available materials without the use of specialized mixing or processing requirements. A specific application was chosen to determine the working range of the intended fiber sensor ( $<30 \%$ strain). Then, sensors were produced by one dip-coating procedure with varying amounts of conductive carbon black to determine the percolation threshold and the performance with respect to carbon black loading. The $50 \mathrm{wt} \%$ carbon black performed significantly better than lower carbon black loadings with respect to linear response and hysteresis. The piezoresistive sheath was applied to two cores with different mechanical properties to determine the supportive core's effect on the sensor's performance. Better adhesion of the sheath to an H3078 core allowed higher accessible sensing limits, while the PEU core sensor had lower first cycle hysteresis and a more stable resistance signal. Conditioning the sensors to $40 \%$ strain $(10 \%$ above the intended working region) resulted in sensors without first cycle hysteresis and a stable baseline resistance. To determine the sensor's performance prior to use in prototype devices intended for accurate three-dimensional kinematic movement tracking, they were analyzed using a random wave pattern on a linear stage. We were able to track strain with the piezoresistance signal to a NRMSE of $6.3 \%$ with conditioned sensors using a simple normalization procedure. While we were aware of the limitations of the sensor with respect to rate-dependent behavior causing signal compression, we were able to overcome the inaccuracies of the sensor by utilizing machine learning algorithms to decrease the error of the strain tracking to $1.6 \%$ NRMSE. Then, we developed a smart textile system using the H3078 core fiber strain sensor for measuring trunk three-dimensional kinematic angles. Using random forest regressor, this system detected trunk flexion, rotation, and lateral bending angles with $4.26^{\circ}, 3.52^{\circ}$, and $3.4^{\circ}$ errors, respectively. This showed the feasibility of a smart textile system using our developed sensor for measuring the kinematic data of complicated multiaxial movements. This system could be useful for developing alternatives for the standard kinematic motion-tracking devices composed of IMUs and motion-capture devices that are bulky, non-portable, spatially limited, and susceptible to external magnetic distortions.

Supplementary Materials: The following are available online at http://www.mdpi.com/1424-8220/19/19/4288/s1, Figure S1: Linear stage set up; Figure S2: 30, 40, and $50 \mathrm{wt} \%$ carbon black loading in J3078 trapezoidal wave pattern at 10, 20, and 30\% strain.; Figure S3: SEM images of 40, 80, and 120\% strain profiles for H3078 sensors; Figure S4: Frequency dependent behavior of H3078-core sensors strain from $0-10 \%$ using a sinusoidal wave pattern A) $1 \mathrm{Hx}, \mathrm{B}) 0.1 \mathrm{~Hz}$; Figure S5: Unnormalized change of strain sensors voltage while performing 3 different types of movement; Table S1: Performance results of the algorithm in the detection of 3 angles of flexion, rotatation, and lateral bending for each participant; Figure S6: Exemplary comparison between the principle reference and estimated flexion, rotation, and lateral bending angles in uniaxial movements. 
Author Contributions: Conceptualization, T.J.C., A.R., and C.M.; methodology, T.J.C., A.R.; material production, T.J.C.; software, T.J.C., A.R., and M.G.; formal analysis, T.J.C., A.R., M.G., and C.M.; writing-original draft preparation, T.J.C. and A.R.; writing—review and editing, T.J.C., A.R., M.G., and C.M.; visualization, T.J.C. and A.R.; supervision, C.M.; funding acquisition, C.M.

Funding: This research was funded by the Natural Sciences and Engineering Research Council of Canada (NSERC).

Conflicts of Interest: The authors declare no conflict of interest.

\section{References}

1. Stoppa, M.; Chiolerio, A. Wearable Electronics and Smart Textiles: A Critical Review. Sensors 2014, 14, 11957-11992. [CrossRef] [PubMed]

2. Pu, X.; Li, L.; Liu, M.; Jiang, C.; Du, C.; Zhao, Z.; Hu, W.; Wang, Z.L. Wearable Self-Charging Power Textile Based on Flexible Yarn Supercapacitors and Fabric Nanogenerators. Adv. Mater. 2015, 1, 98-105. [CrossRef]

3. Nilsson, E.; Mateu, L.; Spies, P.; Hagström, B. Energy Harvesting from Piezoelectric Textile Fibers. Procedia Eng. 2014, 87, 1569-1572. [CrossRef]

4. Lund, A.; Rundqvist, K.; Nilsson, E.; Yu, L.; Hagström, B.; Müller, C. Energy Harvesting Textiles for a Rainy Day: Woven Piezoelectrics Based on Melt-Spun PVDF Microfibres with a Conducting Core. NPJ Flex. Electron. 2018, 2, 9. [CrossRef]

5. Bahk, J.-H.; Fang, H.; Yazawa, K.; Shakouri, A. Flexible Thermoelectric Materials and Device Optimization for Wearable Energy Harvesting. J. Mater. Chem. C 2015, 3, 10362-10374. [CrossRef]

6. Peng, H. Fiber-Shaped Energy Harvesting and Storage Devices; Nanostructure Science and Technology; Springer: Berlin, Germany; Heidelberg: New York, NY, USA, 2015. [CrossRef]

7. Cai, X.; Peng, M.; Yu, X.; Fu, Y.; Zou, D. Flexible Planar/Fiber-Architectured Supercapacitors for Wearable Energy Storage. J. Mater. Chem. C 2014, 2, 1184-1200. [CrossRef]

8. Meng, Y.; Zhao, Y.; Hu, C.; Cheng, H.; Hu, Y.; Zhang, Z.; Shi, G.; Qu, L. All-Graphene Core-Sheath Microfibers for All-Solid-State, Stretchable Fibriform Supercapacitors and Wearable Electronic Textiles. Adv. Mater. 2013, 25, 2326-2331. [CrossRef]

9. Harrison, D.; Qiu, F.; Fyson, J.; Xu, Y.; Evans, P.; Southee, D. A Coaxial Single Fibre Supercapacitor for Energy Storage. Phys. Chem. Chem. Phys. 2013, 15, 12215-12219. [CrossRef]

10. Bae, J.; Song, M.K.; Park, Y.J.; Kim, J.M.; Liu, M.; Wang, Z.L. Fiber Supercapacitors Made of Nanowire-Fiber Hybrid Structures for Wearable/Flexible Energy Storage. Angew. Chem. Int. Ed. 2011, 50, 1683-1687. [CrossRef]

11. Castano, L.M.; Flatau, A.B. Smart Fabric Sensors and E-Textile Technologies: A Review. Smart Mater. Struct. 2014, 23, 53001-53027. [CrossRef]

12. Yamada, T.; Hayamizu, Y.; Yamamoto, Y.; Yomogida, Y.; Izadi-Najafabadi, A.; Futaba, D.N.; Hata, K. A Stretchable Carbon Nanotube Strain Sensor for Human-Motion Detection. Nat. Nanotechnol. 2011, 6, $296-301$. [CrossRef] [PubMed]

13. Kim, S.-R.; Kim, J.-H.; Park, J.-W. Wearable and Transparent Capacitive Strain Sensor with High Sensitivity Based on Patterned Ag Nanowire Networks. ACS Appl. Mater. Interfaces 2017, 9, 26407-26416. [CrossRef] [PubMed]

14. Amjadi, M.; Kyung, K.-U.; Park, I.; Sitti, M. Stretchable, Skin-Mountable, and Wearable Strain Sensors and Their Potential Applications: A Review. Adv. Funct. Mater. 2016, 26, 1678-1698. [CrossRef]

15. Zhou, Y.; He, J.; Wang, H.; Qi, K.; Nan, N.; You, X.; Shao, W.; Wang, L.; Ding, B.; Cui, S. Highly Sensitive, Self-Powered and Wearable Electronic Skin Based on Pressure-Sensitive Nanofiber Woven Fabric Sensor. Sci. Rep. 2017, 7, 12949-12958. [CrossRef] [PubMed]

16. Chhetry, A.; Yoon, H.; Park, J.Y. A Flexible and Highly Sensitive Capacitive Pressure Sensor Based on Conductive Fibers with a Microporous Dielectric for Wearable Electronics. J. Mater. Chem. C. 2017, 5, 10068-10076. [CrossRef]

17. Lee, J.; Kwon, H.; Seo, J.; Shin, S.; Koo, J.H.; Pang, C.; Son, S.; Kim, J.H.; Jang, Y.H.; Kim, D.E.; et al. Conductive Fiber-Based Ultrasensitive Textile Pressure Sensor for Wearable Electronics. Adv. Mater. 2015, 27, $2433-2439$. [CrossRef] 
18. Huang, C.; Soenen, S.J.; Rejman, J.; Lucas, B.; Braeckmans, K.; Demeester, J.; De Smedt, S.C. Stimuli-Responsive Electrospun Fibers and Their Applications. Chem. Soc. Rev. 2011, 40, 2417-2434. [CrossRef] [PubMed]

19. Needham, R.; Stebbins, J.; Chockalingam, N. Three-Dimensional Kinematics of the Lumbar Spine during Gait Using Marker-Based Systems: A Systematic Review. J. Med. Eng. Tech. 2016, 40, 172-185. [CrossRef]

20. Lebel, K.; Boissy, P.; Hamel, M.; Duval, C. Inertial Measures of Motion for Clinical Biomechanics: Comparative Assessment of Accuracy under Controlled Conditions-Effect of Velocity. PLoS ONE 2013, 8, e79945. [CrossRef]

21. Dehzangi, O.; Taherisadr, M.; ChangalVala, R. IMU-Based Gait Recognition Using Convolutional Neural Networks and Multi-Sensor Fusion. Sensors 2017, 17, 2735. [CrossRef]

22. Choi, S.; Shin, Y.B.; Kim, S.-Y.; Kim, J. A Novel Sensor-Based Assessment of Lower Limb Spasticity in Children with Cerebral Palsy. J. Neuroeng. Rehabil. 2018, 15, 45. [CrossRef] [PubMed]

23. Tafazzol, A.; Arjmand, N.; Shirazi-Adl, A.; Parnianpour, M. Lumbopelvic Rhythm during Forward and Backward Sagittal Trunk Rotations: Combined In Vivo Measurement with Inertial Tracking Device and Biomechanical Modeling. Clin. Biomech. 2014, 29, 7-13. [CrossRef] [PubMed]

24. Hajibozorgi, M.; Arjmand, N. Sagittal Range of Motion of the Thoracic Spine Using Inertial Tracking Device and Effect of Measurement Errors on Model Predictions. J. Biomech. 2016, 49, 913-918. [CrossRef] [PubMed]

25. Intolo, P.; Carman, A.B.; Milosavljevic, S.; Abbott, J.H.; Baxter, G.D. The Spineangel ${ }^{\circledR}$ : Examining the Validity and Reliability of a Novel Clinical Device for Monitoring Trunk Motion. Man. Ther. 2010, 15, 160-166. [CrossRef]

26. Kim, S.; Nussbaum, M.A. Performance Evaluation of a Wearable Inertial Motion Capture System for Capturing Physical Exposures during Manual Material Handling Tasks. Ergonomics 2013, 56, 314-326. [CrossRef] [PubMed]

27. Robert-Lachaine, X.; Mecheri, H.; Larue, C.; Plamondon, A. Validation of Inertial Measurement Units with an Optoelectronic System for Whole-Body Motion Analysis. Med. Biol. Eng. Comput. 2017, 55, 609-619. [CrossRef]

28. Godwin, A.; Agnew, M.; Stevenson, J. Accuracy of Inertial Motion Sensors in Static, Quasistatic, and Complex Dynamic Motion. J. Biomech. Eng. 2009, 131, 114501-114505. [CrossRef] [PubMed]

29. Brodie, M.A.; Walmsley, A.; Page, W. Dynamic Accuracy of Inertial Measurement Units during Simple Pendulum Motion. Comput. Methods Biomech. Biomed. Engin. 2008, 11, 235-242. [CrossRef]

30. Samadani, A.; Lee, A.; Kulić, D. A Spinal Motion Measurement Protocol Utilizing Inertial Sensors Without Magnetometers. In Proceedings of the 40th Annual International Conference of the IEEE Engineering in Medicine and Biology Society (EMBC), Honolulu, HI, USA, 17-21 July 2018; pp. 1-4. [CrossRef]

31. Ha, T.-H.; Saber-Sheikh, K.; Moore, A.P.; Jones, M.P. Measurement of Lumbar Spine Range of Movement and Coupled Motion Using Inertial Sensors-A Protocol Validity Study. Man. Ther. 2013, 18, 87-91. [CrossRef]

32. Bauer, C.M.; Rast, F.M.; Ernst, M.J.; Kool, J.; Oetiker, S.; Rissanen, S.M.; Suni, J.H.; Kankaanpää, M. Concurrent Validity and Reliability of a Novel Wireless Inertial Measurement System to Assess Trunk Movement. J. Electromyogr. Kinesiol. 2015, 25, 782-790. [CrossRef]

33. Cooper, C.B.; Arutselvan, K.; Liu, Y.; Armstrong, D.; Lin, Y.; Khan, M.R.; Genzer, J.; Dickey, M.D. Stretchable Capacitive Sensors of Torsion, Strain, and Touch Using Double Helix Liquid Metal Fibers. Adv. Funct. Mater. 2017, 27, 1605630. [CrossRef]

34. Zahid, M.; Papadopoulou, E.L.; Athanassiou, A.; Bayer, I.S. Strain-Responsive Mercerized Conductive Cotton Fabrics Based on PEDOT:PSS/Graphene. Mater. Des. 2017, 135, 213-222. [CrossRef]

35. Yin, F.; Li, X.; Peng, H.; Li, F.; Yang, K.; Yuan, W. A Highly Sensitive, Multifunctional, and Wearable Mechanical Sensor Based on RGO/Synergetic Fiber Bundles for Monitoring Human Actions and Physiological Signals. Sens. Actuators B Chem. 2019, 285, 179-185. [CrossRef]

36. Liu, Z.F.; Fang, S.; Moura, F.A.; Ding, J.N.; Jiang, N.; Di, J.; Zhang, M.; Lepro, X.; Galvao, D.S.; Haines, C.S.; et al. Hierarchically Buckled Sheath-Core Fibers for Superelastic Electronics, Sensors, and Muscles. Science 2015, 349, 400-404. [CrossRef] [PubMed]

37. Mattmann, C.; Clemens, F.; Tröster, G. Sensor for Measuring Strain in Textile. Sensors 2008, 8, 3719-3732. [CrossRef] [PubMed]

38. Seyedin, S.; Razal, J.M.; Innis, P.C.; Jeiranikhameneh, A.; Beirne, S.; Wallace, G.G. Knitted Strain Sensor Textiles of Highly Conductive All-Polymeric Fibers. ACS Appl. Mater. Interfaces 2015, 7, 21150-21158. [CrossRef] 
39. Eom, J.; Jaisutti, R.; Lee, H.; Lee, W.; Heo, J.-S.; Lee, J.-Y.; Park, S.K.; Kim, Y.-H. Highly Sensitive Textile Strain Sensors and Wireless User-Interface Devices Using All-Polymeric Conducting Fibers. ACS Appl. Mater. Interfaces 2017, 9, 10190-10197. [CrossRef]

40. Keulemans, G.; Ceyssens, F.; Puers, R. An Ionic Liquid Based Strain Sensor for Large Displacement Measurement. Biomed. Microdevices 2017, 19, 1-9. [CrossRef]

41. Chen, S.; Liu, H.; Liu, S.; Wang, P.; Zeng, S.; Sun, L.; Liu, L. Transparent and Waterproof Ionic Liquid-Based Fibers for Highly Durable Multifunctional Sensors and Strain-Insensitive Stretchable Conductors. ACS Appl. Mater. Interfaces 2018, 10, 4305-4314. [CrossRef]

42. Nilsson, E.; Oxfall, H.; Wandelt, W.; Rychwalski, R.; Hagström, B. Melt Spinning of Conductive Textile Fibers with Hybridized Graphite Nanoplatelets and Carbon Black Filler. J. Appl. Polym. Sci. 2013, 130, $2579-2587$. [CrossRef]

43. Grigoryev, A.; Sa, V.; Gopishetty, V.; Tokarev, I.; Kornev, K.G.; Minko, S. Wet-Spun Stimuli-Responsive Composite Fibers with Tunable Electrical Conductivity. Adv. Funct. Mater. 2013, 23, 5903-5909. [CrossRef]

44. Tang, Z.; Jia, S.; Wang, F.; Bian, C.; Chen, Y.; Wang, Y.; Li, B. Highly Stretchable Core-Sheath Fibers via Wet-Spinning for Wearable Strain Sensors. ACS Appl. Mater. Interfaces 2018, 10, 6624-6635. [CrossRef] [PubMed]

45. Yoon, J.; Yang, H.-S.; Lee, B.-S.; Yu, W.-R. Recent Progress in Coaxial Electrospinning: New Parameters, Various Structures, and Wide Applications. Adv. Mater. 2017, 30, 1704765. [CrossRef] [PubMed]

46. Labbaf, S.; Ghanbar, H.; Stride, E.; Edirisinghe, M. Preparation of Multilayered Polymeric Structures Using a Novel Four-Needle Coaxial Electrohydrodynamic Device. Macromol. Rapid Commun. 2013, 35, 618-623. [CrossRef] [PubMed]

47. Mahalingam, S.; Homer-Vanniasinkam, S.; Edirisinghe, M. Novel Pressurised Gyration Device for Making Core-Sheath Polymer Fibres. Mater. Des. 2019, 178, 107846. [CrossRef]

48. Heseltine, P.L.; Ahmed, J.; Edirisinghe, M. Developments in Pressurized Gyration for the Mass Production of Polymeric Fibers. Macromol. Mater. Eng. 2018, 303, 1800218. [CrossRef]

49. Medeiros, E.S.; Glenn, G.M.; Klamczynski, A.P.; Orts, W.J.; Mattoso, L.H.C. Solution Blow Spinning: A New Method to Produce Micro- and Nanofibers from Polymer Solutions. J. Appl. Polym. Sci. 2009, 113, 2322-2330. [CrossRef]

50. Gao, Y.; Yu, G.; Shu, T.; Chen, Y.; Yang, W.; Liu, Y.; Long, J.; Xiong, W.; Xuan, F. 3D-Printed Coaxial Fibers for Integrated Wearable Sensor Skin. Adv. Mater. Technol. 2019, 1900504. [CrossRef]

51. Jian, M.; Wang, C.; Wang, Q.; Wang, H.; Xia, K.; Yin, Z.; Zhang, M.; Liang, X.; Zhang, Y. Advanced Carbon Materials for Flexible and Wearable Sensors. Sci. China Mater. 2017, 60, 1026-1062. [CrossRef]

52. Stassi, S.; Cauda, V.; Canavese, G.; Pirri, C.F. Flexible Tactile Sensing Based on Piezoresistive Composites: A Review. Sensors 2014, 14, 5296-5332. [CrossRef]

53. Foroughi, J.; Spinks, G.M.; Aziz, S.; Mirabedini, A.; Jeiranikhameneh, A.; Wallace, G.G.; Kozlov, M.E.; Baughman, R.H. Knitted Carbon-Nanotube-Sheath/Spandex-Core Elastomeric Yarns for Artificial Muscles and Strain Sensing. ACS Nano 2016, 10, 9129-9135. [CrossRef] [PubMed]

54. Montazerian, H.; Rashidi, A.; Dalili, A.; Najjaran, H.; Milani, A.S.; Hoorfar, M. Graphene-Coated Spandex Sensors Embedded into Silicone Sheath for Composites Health Monitoring and Wearable Applications. Small 2019, 15, 1804991. [CrossRef] [PubMed]

55. Chen, S.; Lou, Z.; Chen, D.; Jiang, K.; Shen, G. Polymer-Enhanced Highly Stretchable Conductive Fiber Strain Sensor Used for Electronic Data Gloves. Adv. Mater. Technol. 2016, 1, 1600136. [CrossRef]

56. Zhou, J.; Xu, X.; Xin, Y.; Lubineau, G. Coaxial Thermoplastic Elastomer-Wrapped Carbon Nanotube Fibers for Deformable and Wearable Strain Sensors. Adv. Funct. Mater. 2018, 28, 1705591. [CrossRef]

57. Esfahani, M.I.M.; Nussbaum, M.A. A "Smart" Undershirt for Tracking Upper Body Motions: Task Classification and Angle Estimation. IEEE Sens. J. 2018, 18, 7650-7658. [CrossRef]

58. Yamamoto, A.; Nakamoto, H.; Yamaji, T.; Ootaka, H.; Bessho, Y.; Nakamura, R.; Ono, R. Method for Measuring Tri-Axial Lumbar Motion Angles Using Wearable Sheet Stretch Sensors. PLoS ONE 2017, 12, e0183651. [CrossRef]

59. Breiman, L. Random Forests. Mach. Learn. 2001, 45, 5-32. [CrossRef]

60. Ejupi, A.; Ferrone, A.; Menon, C. Quantification of Textile-Based Stretch Sensors Using Machine Learning: An Exploratory Study. In Proceedings of the 7th IEEE International Conference on Biomedical Robotics and Biomechatronics (Biorob), Enschede, The Netherlands, 26-29 August 2018; pp. 254-259. [CrossRef] 
61. Gholami, M.; Ejupi, A.; Rezaei, A.; Ferrone, A.; Menon, C. Estimation of Knee Joint Angle Using a Fabric-Based Strain Sensor and Machine Learning: A Preliminary Investigation. In Proceedings of the 7th IEEE International Conference on Biomedical Robotics and Biomechatronics (Biorob), Enschede, The Netherlands, 26-29 August 2018; pp. 589-594. [CrossRef]

62. Rezaei, A.; Ejupi, A.; Gholami, M.; Ferrone, A.; Menon, C. Preliminary Investigation of Textile-Based Strain Sensors for the Detection of Human Gait Phases Using Machine Learning. In Proceedings of the 7th IEEE International Conference on Biomedical Robotics and Biomechatronics (Biorob), Enschede, The Netherlands, 26-29 August 2018; pp. 563-568. [CrossRef]

63. Pedregosa, F.; Varoquaux, G.; Gramfort, A.; Michel, V.; Thirion, B.; Grisel, O.; Blondel, M.; Prettenhofer, P.; Weiss, R.; Dubourg, V.; et al. Scikit-Learn: Machine Learning in Python. J. Mach. Learn. Res. 2011, 12, 2825-2830.

64. Mattmann, C. Body Posture Detection Using Strain Sensitive Clothing; ETH: Zürich, Switzerland, 2008.

65. Mattmann, C.; Amft, O.; Harms, H.; Troster, G.; Clemens, F. Recognizing Upper Body Postures Using Textile Strain Sensors. In Proceedings of the 2007 11th IEEE International Symposium on Wearable Computers, Washington, DC, USA, 11-13 October 2007; pp. 29-36. [CrossRef]

66. Consmüller, T.; Rohlmann, A.; Weinland, D.; Schmidt, H.; Zippelius, T.; Duda, G.N.; Taylor, W.R. Automatic Distinction of Upper Body Motions in the Main Anatomical Planes. Med. Eng. Phys. 2014, 36, 516-521. [CrossRef]

67. Tormene, P.; Bartolo, M.; De Nunzio, A.M.; Fecchio, F.; Quaglini, S.; Tassorelli, C.; Sandrini, G. Estimation of Human Trunk Movements by Wearable Strain Sensors and Improvement of Sensor's Placement on Intelligent Biomedical Clothes. Biomed. Eng. Online 2012, 11, 95. [CrossRef]

68. Esfahani, M.I.M.; Nussbaum, M.A.; Kong, Z. Using a Smart Textile System for Classifying Occupational Manual Material Handling Tasks: Evidence from Lab-Based Simulations. Ergonomics 2019, 62, 823-833. [CrossRef] [PubMed]

69. Bersch, S.D.; Azzi, D.; Khusainov, R.; Achumba, I.E.; Ries, J. Sensor Data Acquisition and Processing Parameters for Human Activity Classification. Sensors 2014, 14, 4239-4270. [CrossRef] [PubMed]

70. Bergstrom, J. Constitutive Modeling of the Large Strain Time-Dependent Behavior of Elastomers. J. Mech. Phys. Solids 1998, 46, 931-954. [CrossRef]

71. Le, H.H.; Lüpke, T.; Pham, T.; Radusch, H.-J. Time Dependent Deformation Behavior of Thermoplastic Elastomers. Polymer 2003, 44, 4589-4597. [CrossRef]

72. Yang, H.; Yao, X.; Yuan, L.; Gong, L.; Liu, Y. Strain-Sensitive Electrical Conductivity of Carbon Nanotube-Graphene-Filled Rubber Composites under Cyclic Loading. Nanoscale 2019, 11, 578-586. [CrossRef]

73. Tolvanen, J.; Hannu, J.; Jantunen, H. Stretchable and Washable Strain Sensor Based on Cracking Structure for Human Motion Monitoring. Sci. Rep. 2018, 8. [CrossRef]

74. Roy Choudhury, A.K. Roy Choudhury. Process Control in Finishing of Textiles-Process Control in Textile Manufacturing. In Process in Textile Manufacturing; Woodhead Publishing: Sawston, UK, 2012.

75. Lee, J.; Shin, S.; Lee, S.; Song, J.; Kang, S.; Han, H.; Kim, S.; Kim, S.; Seo, J.; Kim, D.; et al. Highly Sensitive Multifilament Fiber Strain Sensors with Ultrabroad Sensing Range for Textile Electronics. ACS Nano 2018, 12, 4259-4268. [CrossRef]

76. Brown, H.R. The Adhesion between Polymers. Annu. Rev. Mater. Sci. 1991, 21, 463-489. [CrossRef]

77. Kar, K.K.; Bhowmick, A.K. Effect of Holding Time on High Strain Hysteresis Loss of Carbon Black Filled Rubber Vulcanizates. Polym. Eng. Sci. 1998, 38, 1927-1945. [CrossRef]

78. Liao, X.; Wang, W.; Wang, L.; Tang, K.; Zheng, Y. Controllably Enhancing Stretchability of Highly Sensitive Fiber-Based Strain Sensors for Intelligent Monitoring. ACS Appl. Mater. Interfaces 2019, 11, 2431-2440. [CrossRef]

79. Shintake, J.; Piskarev, E.; Jeong, S.H.; Floreano, D. Ultrastretchable Strain Sensors Using Carbon Black-Filled Elastomer Composites and Comparison of Capacitive Versus Resistive Sensors. Adv. Mater. Technol. 2018, 3, 1700284. [CrossRef]

80. Cuesta-Vargas, A.I.; Galán-Mercant, A.; Williams, J.M. The Use of Inertial Sensors System for Human Motion Analysis. Phys. Ther. Rev. 2010, 15, 462-473. [CrossRef] [PubMed]

81. Mokhlespour Esfahani, M.I.; Zobeiri, O.; Moshiri, B.; Narimani, R.; Mehravar, M.; Rashedi, E.; Parnianpour, M. Trunk Motion System (TMS) Using Printed Body Worn Sensor (BWS) via Data Fusion Approach. Sensors 2017, 17, 112. [CrossRef] [PubMed] 
82. Schall, M.C.; Fethke, N.B.; Chen, H.; Oyama, S.; Douphrate, D.I. Accuracy and Repeatability of an Inertial Measurement Unit System for Field-Based Occupational Studies. Ergonomics 2016, 59, 591-602. [CrossRef]

83. de Vries, W.H.K.; Veeger, H.E.J.; Baten, C.T.M.; van der Helm, F.C.T. Magnetic Distortion in Motion Labs, Implications for Validating Inertial Magnetic Sensors. Gait Posture 2009, 29, 535-541. [CrossRef] [PubMed]

84. Abyarjoo, F.; Barreto, A.; Cofino, J.; Ortega, F.R. Implementing a Sensor Fusion Algorithm for 3D Orientation Detection with Inertial/Magnetic Sensors. In Innovations and Advances in Computing, Informatics, Systems Sciences, Networking and Engineering; Sobh, T., Elleithy, K., Eds.; Lecture Notes in Electrical Engineering; Springer International Publishing: New York, NY, USA, 2015; pp. 305-310.

85. Wong, W.Y.; Wong, M.S. Trunk Posture Monitoring with Inertial Sensors. Eur. Spine J. 2008, 17, 743-753. [CrossRef] [PubMed]

(C) 2019 by the authors. Licensee MDPI, Basel, Switzerland. This article is an open access article distributed under the terms and conditions of the Creative Commons Attribution (CC BY) license (http://creativecommons.org/licenses/by/4.0/). 